\title{
New quantum boundaries for quantum Simpson's and quantum Newton's type inequalities for preinvex functions
}

\author{
Muhammad Aamir Ali' ${ }^{1}$, Mujahid Abbas ${ }^{2}$, Hüseyin Budak ${ }^{3}$ Praveen Agarwal ${ }^{4}$, Ghulam Murtaza ${ }^{5}$ and \\ Yu-Ming Chu ${ }^{6 *}$
}

"Correspondence:

chuyuming2005@126.com

${ }^{6}$ Department of Mathematics,

Huzhou University, Huzhou, China

Full list of author information is

available at the end of the article

\section{Springer}

\begin{abstract}
In this research, we derive two generalized integral identities involving the $q^{\varkappa_{2}}$-quantum integrals and quantum numbers, the results are then used to establish some new quantum boundaries for quantum Simpson's and quantum Newton's inequalities for $q$-differentiable preinvex functions. Moreover, we obtain some new and known Simpson's and Newton's type inequalities by considering the limit $q \rightarrow 1^{-}$ in the key results of this paper.
\end{abstract}

Keywords: Simpson's $\frac{1}{3}$ formula; Simpson's $\frac{3}{8}$ formula; Integral inequalities; Quantum calculus; Preinvex functions

\section{Introduction}

In the domain of q-analysis, much work is being carried out initiating from Euler in order to attain adeptness in mathematics that constructs quantum computing q-calculus considered as a relationship between physics and mathematics. In different areas of mathematics, it has numerous applications such as combinatorics, number theory, basic hypergeometric functions, orthogonal polynomials, and other sciences, mechanics, the theory of relativity, and quantum theory [1-5]. Apparently, Euler invented this important mathematics branch. He used the $q$ parameter in Newton's work with infinite series. Later, in a methodical manner, the $q$-calculus that knew without limit calculus was firstly given by Jackson [2]. In 1908-1909, the general form of the $q$-integral and $q$-difference operator is defined by Jackson [4]. In 1969, for the first time Agarwal [6] defined the $q$-fractional derivative. In 1966-1967, Al-Salam [7] introduced a $q$-analog of the $q$-fractional integral and $q$-Riemann-Liouville fractional. In 2004, Rajkovic [8] gave a definition of the Riemanntype $q$-integral which was generalized to Jackson $q$-integral. In 2013, Tariboon introduced $\varkappa_{1} D_{q}$-difference operator [9].

Many integral inequalities are well known in classical analysis, such as the Hölder inequality, Simpson's inequality, Newton's inequality, the Hermite-Hadamard inequality and the Ostrowski inequality, Cauchy-Bunyakovsky-Schwarz, Gruss, Gruss-Cebysev, and other integral inequalities have been proved and applied in the setup of $q$-calculus

(c) The Author(s) 2021. This article is licensed under a Creative Commons Attribution 4.0 International License, which permits use, sharing, adaptation, distribution and reproduction in any medium or format, as long as you give appropriate credit to the original author(s) and the source, provide a link to the Creative Commons licence, and indicate if changes were made. The images or other third party material in this article are included in the article's Creative Commons licence, unless indicated otherwise in a credit line to the material. If material is not included in the article's Creative Commons licence and your intended use is not permitted by statutory regulation or exceeds the permitted use, you will need to obtain permission directly from the copyright holder. To view a copy of this licence, visit http://creativecommons.org/licenses/by/4.0/. 
using classical convexity. Many mathematicians have done studies in $q$-calculus analysis; the interested reader can check [10-24].

Thomas Simpson has developed crucial methods for the numerical integration and estimation of definite integrals considered as Simpson's rule during (1710-1761). Nevertheless, a similar approximation was used by Kepler almost 10 decades earlier, so it is also known as Kepler's rule. Simpson's rule includes the three-point Newton-Cotes quadrature rule, so an estimation based on a three steps quadratic kernel is sometimes called a Newton-type result.

1. Simpson's $1 / 3$ formula is given as

$$
\frac{1}{\varkappa_{2}-\varkappa_{1}} \int_{\varkappa_{1}}^{\varkappa_{2}} \varphi(\varkappa) d \varkappa \approx \frac{1}{6}\left[\varphi\left(\varkappa_{1}\right)+4 \varphi\left(\frac{\varkappa_{1}+\varkappa_{2}}{2}\right)+\varphi\left(\varkappa_{2}\right)\right] .
$$

2. Simpson's $3 / 8$ formula is given as follows

$$
\frac{1}{\varkappa_{2}-\varkappa_{1}} \int_{\varkappa_{1}}^{\varkappa_{2}} \varphi(\varkappa) d \varkappa \approx \frac{1}{8}\left[\varphi\left(\varkappa_{1}\right)+3 \varphi\left(\frac{2 \varkappa_{1}+\varkappa_{2}}{3}\right)+3 \varphi\left(\frac{\varkappa_{1}+2 \varkappa_{2}}{3}\right)+\varphi\left(\varkappa_{2}\right)\right] .
$$

There are a large number of estimations related to these quadrature rules in the literature, one of them is the following estimation known as Simpson's inequality:

Theorem 1 Let $\varphi:\left[\varkappa_{1}, \varkappa_{2}\right] \rightarrow \mathbb{R}$ be a four times continuously differentiable function on $\left(\varkappa_{1}, \varkappa_{2}\right)$, and

$$
\left\|\varphi^{(4)}\right\|_{\infty}=\sup _{\varkappa \in\left(\varkappa 1, \varkappa_{2}\right)}\left|\varphi^{(4)}(\varkappa)\right|<\infty .
$$

Then we have the following inequality:

$$
\begin{aligned}
& \left|\frac{1}{3}\left[\frac{\varphi\left(\varkappa_{1}\right)+\varphi\left(\varkappa_{2}\right)}{2}+2 \varphi\left(\frac{\varkappa_{1}+\varkappa_{2}}{2}\right)\right]-\frac{1}{\varkappa_{2}-\varkappa_{1}} \int_{\varkappa_{1}}^{\varkappa_{2}} \varphi(\varkappa) d \varkappa\right| \\
& \quad \leq \frac{1}{2880}\left\|\varphi^{(4)}\right\|_{\infty}\left(\varkappa_{2}-\varkappa_{1}\right)^{4} .
\end{aligned}
$$

In the recent era, Simpson's type of inequalities has been emphasized by many authors for numerous types of functions. Convexity is useful and potent for solving different problems that appear within various branches of applied and pure mathematics. For an instance, Dragomir demonstrated novel Simpson's type consequences and their applications to quadratic formulas in numerical integration in [25]. Furthermore, Alomari [26] has presented Simpson's type of inequalities for $s$-convex functions. The refinements of Simpson's type of inequalities depending on convexity have been visualized by Sarikaya et al. in [27]. For the further studies of this area, one can consult [28-30].

The main objective of this paper is to study Newton's and Simpson's type inequalities for preinvex functions by using the notions of quantum calculus.

\section{Preliminaries of $q$-calculus and some inequalities}

The basic notions and findings which are needed in the sequel to prove our crucial results are reviewed in this section. Throughout this paper, we assume that $\varkappa_{1}<\varkappa_{2}$ and $0<q<1$. Let $\omega$ be a nonempty closed set in $\mathbb{R}^{n}, \varphi: \omega \rightarrow \mathbb{R}$ be a continuous function, and $\eta(\cdot, \cdot)$ : $\omega \times \omega \rightarrow \mathbb{R}^{n}$ be a continuous bifunction. 
Definition 1 ([13]) A set $\omega$ is called an invex set respecting a bifunction $\eta(\cdot, \cdot)$ if

$$
\varkappa_{2}+t \eta\left(\varkappa_{1}, \varkappa_{2}\right) \in \omega, \quad \forall \varkappa_{1}, \varkappa_{2} \in \omega, t \in[0,1]
$$

The invex set $\omega$ is more commonly referred to as $\eta$-connected set.

Definition 2 ([13]) A mapping $\varphi$ is called a preinvex respecting an arbitrary bifunction $\eta(\cdot, \cdot)$ if the following inequality holds:

$$
\varphi\left(\varkappa_{2}+t \eta\left(\varkappa_{1}, \varkappa_{2}\right)\right) \leq t \varphi\left(\varkappa_{1}\right)+(1-t) \varphi\left(\varkappa_{2}\right), \quad \forall \varkappa_{1}, \varkappa_{2} \in \omega, t \in[0,1] .
$$

The function $\varphi$ is called preconcave if $-\varphi$ is preinvex.

Remark 1 If we set $\eta\left(\varkappa_{1}, \varkappa_{2}\right)=\varkappa_{1}-\varkappa_{2}$, then the definition of preinvex function reduces to the definition of a convex function given below;

$$
\varphi\left(\varkappa_{2}+t\left(\varkappa_{1}-\varkappa_{2}\right)\right) \leq t \varphi\left(\varkappa_{1}\right)+(1-t) \varphi\left(\varkappa_{2}\right), \quad \forall \varkappa_{1}, \varkappa_{2} \in \omega, t \in[0,1] .
$$

Now we present some well-known concepts and theorems for $q$-derivatives and $q$ integrals of a function $\varphi$ on $\left[\varkappa_{1}, \varkappa_{2}\right]$.

Definition $3([5,9])$ We assume that a function $\varphi:\left[\varkappa_{1}, \varkappa_{2}\right] \rightarrow \mathbb{R}$ is continuous. Then the $q_{\varkappa_{1}}$-derivative of $\varphi$ at $\varkappa \in\left[\varkappa_{1}, \varkappa_{2}\right]$ is defined by

$$
\varkappa_{1} D_{q} \varphi(\varkappa)=\frac{\varphi(\varkappa)-\varphi\left(q \varkappa+(1-q) \varkappa_{1}\right)}{(1-q)\left(\varkappa-\varkappa_{1}\right)}, \quad \varkappa \neq \varkappa_{1} .
$$

Since $\varphi:\left[\varkappa_{1}, \varkappa_{2}\right] \rightarrow \mathbb{R}$ is a continuous function, we can define

$$
\varkappa_{1} D_{q} \varphi\left(\varkappa_{1}\right)=\lim _{\varkappa \rightarrow \varkappa_{1}} D_{q} \varphi(\varkappa) .
$$

The function $\varphi$ is called $q_{\varkappa_{1}}$-differentiable on $\left[\varkappa_{1}, \varkappa_{2}\right]$ if $\varkappa_{1} D_{q} \varphi(\varkappa)$ exists for all $\varkappa \in$ $\left[\varkappa_{1}, \varkappa_{2}\right]$. If we assume $\varkappa_{1}=0$ in $(2.1)$, then ${ }_{0} D_{q} \varphi(\varkappa)=D_{q} \varphi(\varkappa)$, where $D_{q} \varphi(\varkappa)$ is the familiar $q$-derivative of $\varphi$ at $\varkappa \in\left[0, \varkappa_{2}\right]$ defined as follows (see [5]):

$$
D_{q} \varphi(\varkappa)=\frac{\varphi(\varkappa)-\varphi(q \varkappa)}{(1-q) \varkappa}, \quad \varkappa \neq 0 .
$$

Definition 4 ([31]) We assume that a function $\varphi:\left[\varkappa_{1}, \varkappa_{2}\right] \rightarrow \mathbb{R}$ is continuous. Then the $q_{\varkappa_{1}}$-definite integral on $\left[\varkappa_{1}, \varkappa_{2}\right]$ is defined by

$$
\int_{\varkappa_{1}}^{\varkappa} \varphi(t)_{\varkappa_{1}} d_{q} t=(1-q)\left(\varkappa-\varkappa_{1}\right) \sum_{n=0}^{\infty} q^{n} \varphi\left(q^{n} \varkappa+\left(1-q^{n}\right) \varkappa_{1}\right), \quad \varkappa \in\left[\varkappa_{1}, \varkappa_{2}\right] .
$$

Remark 2 If $\varkappa_{1}=0$ in (2.3), then $\int_{0}^{\varkappa} \varphi(t)_{0} d_{q} t=\int_{0}^{\varkappa} \varphi(t) d_{q} t$, where $\int_{0}^{\varkappa} \varphi(t) d_{q} t$ is the familiar $q$-definite integral (see, [5]) on $[0, \varkappa]$ defined by

$$
\int_{0}^{\varkappa} \varphi(t)_{0} d_{q} t=\int_{0}^{\varkappa} \varphi(t) d_{q} t=(1-q) \varkappa \sum_{n=0}^{\infty} q^{n} \varphi\left(q^{n} \varkappa\right) .
$$


Definition 5 If $c \in\left(\varkappa_{1}, \varkappa\right)$, then the $q$-definite integral on $[c, \varkappa]$ is expressed as

$$
\int_{c}^{\varkappa} \varphi(t)_{\varkappa_{1}} d_{q} t=\int_{\varkappa_{1}}^{\varkappa} \varphi(t)_{\varkappa_{1}} d_{q} t-\int_{\varkappa_{1}}^{c} \varphi(t)_{\varkappa_{1}} d_{q} t
$$

Alp et al. [9] proved the following $q$-Hermite-Hadamard inequality:

Theorem 2 ( $q_{a}$-Hermite-Hadamard inequality) We assume that a function $\varphi:\left[\varkappa_{1}, \varkappa_{2}\right] \rightarrow$ $\mathbb{R}$ is convex differentiable on $\left[\varkappa_{1}, \varkappa_{2}\right]$ and $0<q<1$. Then we have the following inequality:

$$
\varphi\left(\frac{q \varkappa_{1}+\varkappa_{2}}{[2]_{q}}\right) \leq \frac{1}{\varkappa_{2}-\varkappa_{1}} \int_{\varkappa_{1}}^{\varkappa_{2}} \varphi\left(\varkappa \varkappa_{\varkappa_{1}} d_{q} \varkappa \leq \frac{q \varphi\left(\varkappa_{1}\right)+\varphi\left(\varkappa_{2}\right)}{[2]_{q}} .\right.
$$

On the other hand, Bermudo et al. [32] gave the following new definitions of a quantum integral and derivative. In the same paper, the authors proved a new variant of the quantum Hermite-Hadamard type inequality linked to their newly defined quantum integral.

Definition 6 ([32]) Let $\varphi:\left[\varkappa_{1}, \varkappa_{2}\right] \rightarrow \mathbb{R}$ be a continuous function. Then the $q^{\varkappa_{2}}$-definite integral on $\left[\varkappa_{1}, \varkappa_{2}\right]$ is given by

$$
\begin{aligned}
\int_{\varkappa_{1}}^{\varkappa_{2}} \varphi(\varkappa)^{\varkappa_{2}} d_{q} \varkappa & =(1-q)\left(\varkappa_{2}-\varkappa_{1}\right) \sum_{n=0}^{\infty} q^{n} \varphi\left(q^{n} \varkappa_{1}+\left(1-q^{n}\right) \varkappa_{2}\right) \\
& =\left(\varkappa_{2}-\varkappa_{1}\right) \int_{0}^{1} \varphi\left(t \varkappa_{1}+(1-t) \varkappa_{2}\right) d_{q} t .
\end{aligned}
$$

Definition 7 ([32]) Let $\varphi:\left[\varkappa_{1}, \varkappa_{2}\right] \rightarrow \mathbb{R}$ be a continuous function. Then the $q^{\varkappa_{2}}$ derivative of $\varphi$ at $\varkappa \in\left[\varkappa_{1}, \varkappa_{2}\right]$ is given by

$$
\varkappa_{2} D_{q} \varphi(\varkappa)=\frac{\varphi\left(q \varkappa+(1-q) \varkappa_{2}\right)-\varphi(\varkappa)}{(1-q)\left(\varkappa_{2}-\varkappa\right)}, \quad \varkappa \neq \varkappa_{2} .
$$

Theorem 3 ( $q^{b}$-Hermite-Hadamard inequality, [32]) We consider that a function $\varphi$ : $\left[\varkappa_{1}, \varkappa_{2}\right] \rightarrow \mathbb{R}$ is convex differentiable on $\left[\varkappa_{1}, \varkappa_{2}\right]$ and $0<q<1$. Then we have the following inequality:

$$
\varphi\left(\frac{\varkappa_{1}+q \varkappa_{2}}{[2]_{q}}\right) \leq \frac{1}{\varkappa_{2}-\varkappa_{1}} \int_{\varkappa_{1}}^{\varkappa_{2}} \varphi(\varkappa)^{\varkappa_{2}} d_{q} \varkappa \leq \frac{\varphi\left(\varkappa_{1}\right)+q \varphi\left(\varkappa_{2}\right)}{[2]_{q}} .
$$

Let us set the following notations:

$$
[n]_{q}= \begin{cases}\frac{q^{n}-1}{q-1}=\sum_{i=0}^{n-1} q^{i}, & n \in N, \\ \frac{q^{n}-1}{q-1}, & n \in C,\end{cases}
$$

and

$$
(1-t)_{q}^{n}=(t, q)_{n}=\prod_{i=0}^{n-1}\left(1-q^{i} t\right)
$$


Lemma 1 ([9]) For $a \in \mathbb{R} \backslash\{-1\}$, the following formula holds:

$$
\int_{\varkappa_{1}}^{\varkappa}\left(t-\varkappa_{1}\right)^{a} \varkappa_{1} d_{q} t=\frac{\left(\varkappa-\varkappa_{1}\right)^{a+1}}{[a+1]_{q}} .
$$

\section{Quantum integral identities}

In this section, we will prove two equalities which will help us to obtain our main results.

Lemma 2 Let $\varphi: I=\left[\varkappa_{2}+\eta\left(\varkappa_{1}, \varkappa_{2}\right), \varkappa_{2}\right] \rightarrow \mathbb{R}$ be a $q^{\varkappa_{2}}$-differentiable function on I with $-\eta\left(\varkappa_{1}, \varkappa_{2}\right)=\eta\left(\varkappa_{2}, \varkappa_{1}\right)>0$ and $0<q<1$. If $\varkappa_{2} D_{q} \varphi$ is continuous and integrable on $I$, then the following identity holds for $q^{\varkappa_{2}}$-integrals:

$$
\begin{aligned}
& \frac{1}{\eta\left(\varkappa_{2}, \varkappa_{1}\right)} \int_{\varkappa_{2}+\eta\left(\varkappa_{1}, \varkappa_{2}\right)}^{\varkappa_{2}} \varphi\left(\varkappa^{\varkappa_{2}} d_{q} \varkappa\right. \\
& \quad-\frac{1}{[6]_{q}}\left[\varphi\left(\varkappa_{2}+\eta\left(\varkappa_{1}, \varkappa_{2}\right)\right)+[4]_{q} q^{2} \varphi\left(\frac{[2]_{q} \varkappa_{2}+\eta\left(\varkappa_{1}, \varkappa_{2}\right)}{[2]_{q}}\right)+q \varphi\left(\varkappa_{2}\right)\right] \\
& \quad=q \eta\left(\varkappa_{2}, \varkappa_{1}\right) \int_{0}^{1} \Psi_{q}(t)^{\varkappa_{2}} D_{q} \varphi\left(\varkappa_{2}+t \eta\left(\varkappa_{1}, \varkappa_{2}\right)\right) d_{q} t,
\end{aligned}
$$

where

$$
\Psi_{q}(t)= \begin{cases}t-\frac{1}{[6]_{q}} & \text { if } t \in\left[0, \frac{1}{[2]_{q}}\right), \\ t-\frac{[5]_{q}}{[6]_{q}} & \text { if } t \in\left[\frac{1}{[2]_{q}}, 1\right] .\end{cases}
$$

Proof Using the basic properties of $q$-integral and the definition of $\Psi_{q}(t)$, we have

$$
\begin{aligned}
& \int_{0}^{1} \Psi_{q}(t)^{\varkappa_{2}} D_{q} \varphi\left(\varkappa_{2}+t \eta\left(\varkappa_{1}, \varkappa_{2}\right)\right) d_{q} t
\end{aligned}
$$

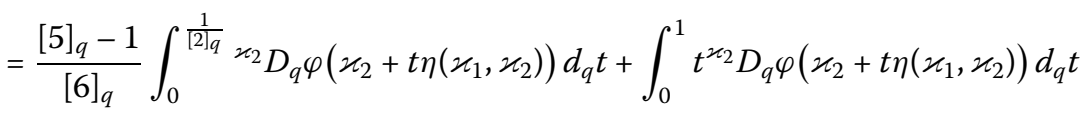

$$
\begin{aligned}
& -\frac{[5]_{q}}{[6]_{q}} \int_{0}^{1} \varkappa_{2} D_{q} \varphi\left(\varkappa_{2}+\operatorname{t\eta }\left(\varkappa_{1}, \varkappa_{2}\right)\right) d_{q} t \text {. }
\end{aligned}
$$

From Definition 7, we have

$$
{ }^{\varkappa_{2}} D_{q} \varphi\left(\varkappa_{2}+\operatorname{t\eta }\left(\varkappa_{1}, \varkappa_{2}\right)\right)=\frac{\varphi\left(\varkappa_{2}+t q \eta\left(\varkappa_{1}, \varkappa_{2}\right)\right)-\varphi\left(\varkappa_{2}+t \eta\left(\varkappa_{1}, \varkappa_{2}\right)\right)}{(1-q) \operatorname{t\eta }\left(\varkappa_{2}, \varkappa_{1}\right)} .
$$

Now, we compute the integrals on the right side of (3.2). Using Definition 6, we obtain

$$
\begin{aligned}
& \int_{0}^{\frac{1}{[2] q}} \varkappa_{2} D_{q} \varphi\left(\varkappa_{2}+t \eta\left(\varkappa_{1}, \varkappa_{2}\right)\right) d_{q} t \\
& =\int_{0}^{\frac{1}{[2]_{q}}} \frac{\varphi\left(\varkappa_{2}+\operatorname{tq\eta }\left(\varkappa_{1}, \varkappa_{2}\right)\right)-\varphi\left(\varkappa_{2}+t \eta\left(\varkappa_{1}, \varkappa_{2}\right)\right)}{(1-q) \operatorname{t\eta }\left(\varkappa_{2}, \varkappa_{1}\right)} d_{q} t \\
& =\frac{1}{\eta\left(\varkappa_{2}, \varkappa_{1}\right)}\left[\sum_{n=0}^{\infty} \varphi\left(\varkappa_{2}+\frac{q^{n+1}}{[2]_{q}} \eta\left(\varkappa_{1}, \varkappa_{2}\right)\right)-\sum_{n=0}^{\infty} \varphi\left(\varkappa_{2}+\frac{q^{n}}{[2]_{q}} \eta\left(\varkappa_{1}, \varkappa_{2}\right)\right)\right]
\end{aligned}
$$




$$
\begin{aligned}
& =\frac{1}{\eta\left(\varkappa_{2}, \varkappa_{1}\right)}\left[\varphi\left(\varkappa_{2}\right)-\varphi\left(\frac{[2]_{q} \varkappa_{2}+\eta\left(\varkappa_{1}, \varkappa_{2}\right)}{[2]_{q}}\right)\right], \\
& \int_{0}^{1} \varkappa_{2} D_{q} \varphi\left(\varkappa_{2}+t \eta\left(\varkappa_{1}, \varkappa_{2}\right)\right) d_{q} t=\frac{1}{\eta\left(\varkappa_{2}, \varkappa_{1}\right)}\left[\varphi\left(\varkappa_{2}\right)-\varphi\left(\varkappa_{2}+\eta\left(\varkappa_{1}, \varkappa_{2}\right)\right)\right],
\end{aligned}
$$

and

$$
\begin{aligned}
& \int_{0}^{1} t^{\varkappa_{2}} D_{q} \varphi\left(\varkappa_{2}+t \eta\left(\varkappa_{1}, \varkappa_{2}\right)\right) d_{q} t \\
& \quad=\int_{0}^{1} \frac{\varphi\left(\varkappa_{2}+t q \eta\left(\varkappa_{1}, \varkappa_{2}\right)\right)-\varphi\left(\varkappa_{2}+t \eta\left(\varkappa_{1}, \varkappa_{2}\right)\right)}{(1-q) \eta\left(\varkappa_{2}, \varkappa_{1}\right)} d_{q} t \\
& \quad=\frac{1}{\eta\left(\varkappa_{2}, \varkappa_{1}\right)}\left[\frac{1-q}{q} \sum_{n=0}^{\infty} q^{n} \varphi\left(\varkappa_{2}+q^{n} \eta\left(\varkappa_{1}, \varkappa_{2}\right)\right)-\frac{\varphi\left(\varkappa_{2}+\eta\left(\varkappa_{1}, \varkappa_{2}\right)\right)}{q}\right] \\
& \quad=\frac{1}{\eta\left(\varkappa_{2}, \varkappa_{1}\right)}\left[\frac{1}{q \eta\left(\varkappa_{2}, \varkappa_{1}\right)} \int_{\varkappa_{2}+\eta\left(\varkappa_{1}, \varkappa_{2}\right)}^{\varkappa_{2}} \varphi\left(\varkappa^{\varkappa_{2}} d_{q} \varkappa_{-} \frac{\varphi\left(\varkappa_{2}+\eta\left(\varkappa_{1}, \varkappa_{2}\right)\right)}{q}\right] .\right.
\end{aligned}
$$

Finally, by substituting (3.3)-(3.5) in (3.2) and multiplying the resultant equality by $\eta\left(\varkappa_{2}, \varkappa_{1}\right)$, we obtain the required identity, which completes the proof.

Remark 3 If we set $\eta\left(\varkappa_{2}, \varkappa_{1}\right)=\varkappa_{2}-\varkappa_{1}$ and $\eta\left(\varkappa_{1}, \varkappa_{2}\right)=\varkappa_{1}-\varkappa_{2}$ in Lemma 2 , then we have the following identity:

$$
\begin{aligned}
& \frac{1}{\left(\varkappa_{2}-\varkappa_{1}\right)} \int_{\varkappa_{1}}^{\varkappa_{2}} \varphi(t)^{\varkappa_{2}} d_{q} t-\frac{1}{[6]_{q}}\left[\varphi\left(\varkappa_{1}\right)+q^{2}[4]_{q} \varphi\left(\frac{\varkappa_{1}+q \varkappa_{2}}{[2]_{q}}\right)+q \varphi\left(\varkappa_{2}\right)\right] \\
& =q\left(\varkappa_{2}-\varkappa_{1}\right) \int_{0}^{1} \Psi_{q}(t)^{\varkappa_{2}} D_{q} \varphi\left(t \varkappa_{1}+(1-t) \varkappa_{2}\right) d_{q} t,
\end{aligned}
$$

where

$$
\Psi_{q}(t)= \begin{cases}t-\frac{1}{[6]_{q}} & \text { if } t \in\left[0, \frac{1}{[2]_{q}}\right), \\ t-\frac{[5]_{q}}{[6]_{q}} & \text { if } t \in\left[\frac{1}{[2]_{q}}, 1\right],\end{cases}
$$

which is given by Ali et al. in [33].

Lemma 3 Let $\varphi: I=\left[\varkappa_{2}+\eta\left(\varkappa_{1}, \varkappa_{2}\right), \varkappa_{2}\right] \rightarrow \mathbb{R}$ be a $q^{\varkappa_{2}}$-differentiable function on I with $-\eta\left(\varkappa_{1}, \varkappa_{2}\right)=\eta\left(\varkappa_{2}, \varkappa_{1}\right)>0$ and $0<q<1$. If $\varkappa_{2} D_{q} \varphi$ is continuous and integrable on $I$, then one has the identity

$$
\begin{aligned}
& \frac{1}{\eta\left(\varkappa_{2}, \varkappa_{1}\right)} \int_{\varkappa_{2}+\eta\left(\varkappa_{1}, \varkappa_{2}\right)}^{\varkappa_{2}} \varphi\left(\varkappa^{\varkappa_{2}} d_{q} \varkappa^{3}\right. \\
& \quad-\frac{1}{[8]_{q}}\left[\varphi\left(\varkappa_{2}+\eta\left(\varkappa_{1}, \varkappa_{2}\right)\right)+\frac{q^{3}[6]_{q}}{[2]_{q}} \varphi\left(\frac{[3]_{q} \varkappa_{2}+\eta\left(\varkappa_{1}, \varkappa_{2}\right)}{[3]_{q}}\right)\right. \\
& \left.\quad+\frac{q^{2}[6]_{q}}{[2]_{q}} \varphi\left(\frac{[3]_{q} \varkappa_{2}+[2]_{q} \eta\left(\varkappa_{1}, \varkappa_{2}\right)}{[3]_{q}}\right)+q \varphi\left(\varkappa_{2}\right)\right] \\
& =q \eta\left(\varkappa_{2}, \varkappa_{1}\right) \int_{0}^{1} \Delta_{q}(t)^{\varkappa_{2}} D_{q} \varphi\left(\varkappa_{2}+t \eta\left(\varkappa_{1}, \varkappa_{2}\right)\right) d_{q} t,
\end{aligned}
$$


where

$$
\Delta_{q}(t)= \begin{cases}t-\frac{1}{[8]_{q}} & \text { if } t \in\left[0, \frac{1}{[3]_{q}}\right), \\ t-\frac{1}{[2]_{q}} & \text { if } t \in\left[\frac{1}{[3]_{q}}, \frac{[2]_{q}}{[3]_{q}}\right), \\ t-\frac{[7]_{q}}{[8]_{q}} & \text { if } t \in\left[\frac{[2]_{q}}{[3]_{q}}, 1\right] .\end{cases}
$$

Proof By the fundamental properties of $q$-integrals and the definition of $\Delta_{q}(t)$, we obtain

$$
\begin{aligned}
& \int_{0}^{1} \Delta_{q}(t)^{\varkappa_{2}} D_{q}\left(\varkappa_{2}+t \eta\left(\varkappa_{1}, \varkappa_{2}\right)\right) d_{q} t \\
& =\frac{[8]_{q}-[2]_{q}}{[8]_{q}[2]_{q}} \int_{0}^{\frac{1}{[3]_{q}}} \varkappa_{2} D_{q} \varphi\left(\varkappa_{2}+t \eta\left(\varkappa_{1}, \varkappa_{2}\right)\right) d_{q} t \\
& +\frac{[7]_{q}[2]_{q}-[8]_{q}}{[8]_{q}[2]_{q}} \int_{0}^{\left[\frac{[2]_{q}}{[]_{q}} \varkappa_{2}\right.} D_{q} \varphi\left(\varkappa_{2}+t \eta\left(\varkappa_{1}, \varkappa_{2}\right)\right) d_{q} t \\
& +\int_{0}^{1}\left(t-\frac{[7]_{q}}{[8]_{q}}\right) \varkappa_{2} D_{q} \varphi\left(\varkappa_{2}+t \eta\left(\varkappa_{1}, \varkappa_{2}\right)\right) d_{q} t .
\end{aligned}
$$

Following arguments similar to those in the proof of Lemma 2, the required identity can be proved.

Remark 4 If we set $\eta\left(\varkappa_{2}, \varkappa_{1}\right)=\varkappa_{2}-\varkappa_{1}$ and $\eta\left(\varkappa_{1}, \varkappa_{2}\right)=\varkappa_{1}-\varkappa_{2}$ in Lemma 3, then we have the following identity:

$$
\begin{aligned}
& \frac{1}{\left(\varkappa_{2}-\varkappa_{1}\right)} \int_{\varkappa_{1}}^{\varkappa_{2}} \varphi(t)^{\varkappa_{2}} d_{q} t \\
& \quad-\frac{1}{[8]_{q}}\left[\varphi\left(\varkappa_{1}\right)+\frac{q^{3}[6]_{q}}{[2]_{q}} \varphi\left(\frac{\varkappa_{1}+q[2]_{q} \varkappa_{2}}{[3]_{q}}\right)+\frac{q^{2}[6]_{q}}{[2]_{q}} \varphi\left(\frac{[2]_{q} \varkappa_{1}+q^{2} \varkappa_{2}}{[3]_{q}}\right)+q \varphi\left(\varkappa_{2}\right)\right] \\
& =q\left(\varkappa_{2}-\varkappa_{1}\right) \int_{0}^{1} \Delta_{q}(t)^{\varkappa_{2}} D_{q} \varphi\left(t \varkappa_{1}+(1-t) \varkappa_{2}\right) d_{q} t
\end{aligned}
$$

where

$$
\Delta_{q}(t)= \begin{cases}t-\frac{1}{[8]_{q}} & \text { if } t \in\left[0, \frac{1}{[3]_{q}}\right) \\ t-\frac{1}{[2]_{q}} & \text { if } t \in\left[\frac{1}{[3]_{q}}, \frac{[2]_{q}}{[3]_{q}}\right), \\ t-\frac{[7]_{q}}{[8]_{q}} & \text { if } t \in\left[\frac{[2]_{q}}{[3]_{q}}, 1\right]\end{cases}
$$

which is proved by Ali et al. in [33].

\section{Main results}

In this section, we present some new Simpson's and Newton's type inequalities for preinvex functions by using Lemma 2 and Lemma 3, respectively. For brevity, we start this section with some notations which will be used in our new results:

$$
A_{1}(q)=\frac{2 q^{2}[2]_{q}^{2}+[6]_{q}^{2}\left([6]_{q}-[3]_{q}\right)}{[2]_{q}^{3}[3]_{q}[6]_{q}^{3}},
$$




$$
\begin{aligned}
B_{1}(q)= & 2 \frac{q[3]_{q}[6]_{q}-q^{2}}{[2]_{q}[3]_{q}[6]_{q}^{3}}+\frac{1}{[2]_{q}^{3}}\left(\frac{q+q^{2}}{[3]_{q}}-\frac{q^{2}+2 q}{[6]_{q}}\right), \\
A_{2}(q)= & \frac{2 q^{2}[5]_{q}^{3}}{[2]_{q}[3]_{q}[6]_{q}^{3}}+\frac{[6]_{q}\left(1+[2]_{q}^{3}\right)-[3]_{q}[5]_{q}\left(1+[2]_{q}^{2}\right)}{[2]_{q}^{3}[3]_{q}[6]_{q}}, \\
B_{2}(q)= & 2 \frac{q[5]_{q}^{2}[6]_{q}[3]_{q}-q^{2}[5]_{q}^{3}}{[2]_{q}[3]_{q}[6]_{q}^{3}}+\frac{q^{2}}{[2]_{q}[3]_{q}}-\frac{q[5]_{q}}{[2]_{q}[6]_{q}} \\
& -\frac{1}{[2]_{q}^{3}}\left[\frac{[5]_{q}\left(2 q+q^{2}\right)}{[6]_{q}}-\frac{q+q^{2}}{[3]_{q}}\right] \\
A_{3}(q)= & \frac{2 q^{2}[3]_{q}^{3}+[8]_{q}^{2}\left([8]_{q}[2]_{q}-[3]_{q}^{2}\right)}{[8]_{q}^{3}[3]_{q}^{4}[2]_{q}}, \\
B_{3}(q)= & 2 \frac{q[8]_{q}[3]_{q}-q^{2}}{[8]_{q}^{3}[2]_{q}[3]_{q}}+\frac{[3]_{q}^{2}-[2]_{q}}{[3]_{q}^{4}[2]_{q}} \\
& +\frac{1-[3]_{q}[2]_{q}}{[8]_{q}[3]_{q}^{2}[2]_{q}}, \\
A_{4}(q)= & \frac{2 q^{2}}{[2]_{q}^{4}[3]_{q}}+\frac{[2]_{q}^{2}\left(1+[2]_{q}^{3}\right)-[3]_{q}^{2}\left(1+[2]_{q}^{2}\right)}{[3]_{q}^{4}[2]_{q}^{2}} \\
B_{4}(q)= & \frac{2 q}{[2]_{q}^{3}}-\frac{q}{[3]_{q}^{2}}-\frac{q^{2}}{[3]_{q}^{2}}-A_{4}(q), \\
A_{5}(q)= & \frac{2 q^{2}[7]_{q}^{3}}{[8]_{q}^{3}[2]_{q}[3]_{q}}+\frac{[2]_{q}[8]_{q}\left([2]_{q}^{3}+[3]_{q}^{3}\right)-[7]_{q}[3]_{q}^{2}\left([2]_{q}^{2}+[3]_{q}^{2}\right)}{[3]_{q}^{4}[8]_{q}[2]_{q}},
\end{aligned}
$$

and

$$
\begin{aligned}
B_{5}(q)= & 2 \frac{q[7]_{q}^{2}[8]_{q}[3]_{q}-q^{2}[7]_{q}^{3}}{[8]_{q}^{3}[2]_{q}[3]_{q}}+\frac{q^{2}}{[2]_{q}[3]_{q}}-\frac{q[7]_{q}}{[2]_{q}[8]_{q}} \\
& +\frac{[2]_{q}\left([3]_{q}^{2}-[2]_{q}^{2}\right)}{[3]_{q}^{4}}-\frac{\left(q+q^{2}\right)[7]_{q}[2]_{q}}{[3]_{q}^{2}[8]_{q}}
\end{aligned}
$$

\subsection{Simpson's type inequalities}

In this subsection, we will prove some quantum Simpson's type inequalities for preinvex functions.

Theorem 4 We assume that the conditions of Lemma 2 hold. If $\left.\right|^{\varkappa_{2}} D_{q} \varphi \mid$ is preinvex and integrable on $I$, then the following inequality holds for $q^{\varkappa_{2}}$-integrals:

$$
\begin{aligned}
& \mid \frac{1}{\eta\left(\varkappa_{2}, \varkappa_{1}\right)} \int_{\varkappa_{2}+\eta\left(\varkappa_{1}, \varkappa_{2}\right)}^{\varkappa_{2}} \varphi(\varkappa)^{\varkappa_{2}} d_{q} \varkappa \\
& \quad-\frac{1}{[6]_{q}}\left[\varphi\left(\varkappa_{2}+\eta\left(\varkappa_{1}, \varkappa_{2}\right)\right)+[4]_{q} q^{2} \varphi\left(\frac{[2]_{q} \varkappa_{2}+\eta\left(\varkappa_{1}, \varkappa_{2}\right)}{[2]_{q}}\right)+q \varphi\left(\varkappa_{2}\right)\right] \mid \\
& \quad \leq q \eta\left(\varkappa_{2}, \varkappa_{1}\right)\left\{\left.\right|^{\varkappa_{2}} D_{q} \varphi\left(\varkappa_{1}\right)\left|\left[A_{1}(q)+A_{2}(q)\right]+\right| \varkappa^{\varkappa_{2}} D_{q} \varphi\left(\varkappa_{2}\right) \mid\left[B_{1}(q)+B_{2}(q)\right]\right\},
\end{aligned}
$$

where $0<q<1$ and $A_{1}(q), A_{2}(q), B_{1}(q), B_{2}(q)$ are given as in (4.1)-(4.4), respectively. 
Proof By taking the modulus in Lemma 2 and using the properties of the modulus, we obtain

$$
\begin{aligned}
& \mid \frac{1}{\eta\left(\varkappa_{2}, \varkappa_{1}\right)} \int_{\varkappa_{2}+\eta\left(\varkappa_{1}, \varkappa_{2}\right)}^{\varkappa_{2}} \varphi\left(\varkappa^{\varkappa_{2}} d_{q} \varkappa^{-1}\right. \\
& \quad-\frac{1}{[6]_{q}}\left[\varphi\left(\varkappa_{2}+\eta\left(\varkappa_{1}, \varkappa_{2}\right)\right)+[4]_{q} q^{2} \varphi\left(\frac{[2]_{q} \varkappa_{2}+\eta\left(\varkappa_{1}, \varkappa_{2}\right)}{[2]_{q}}\right)+q \varphi\left(\varkappa_{2}\right)\right] \mid \\
& \leq q \eta\left(\varkappa_{2}, \varkappa_{1}\right) \int_{0}^{\frac{1}{[2]_{q}}}\left|t-\frac{1}{[6]_{q}}\right|\left|\varkappa^{2} D_{q} \varphi\left(\varkappa_{2}+t \eta\left(\varkappa_{1}, \varkappa_{2}\right)\right)\right| d_{q} t \\
& \quad+q \eta\left(\varkappa_{2}, \varkappa_{1}\right) \int_{\frac{1}{[2]_{q}}}^{1}\left|t-\frac{[5]_{q}}{[6]_{q}}\right| \varkappa^{\varkappa_{2}} D_{q} \varphi\left(\varkappa_{2}+t \eta\left(\varkappa_{1}, \varkappa_{2}\right)\right) \mid d_{q} t .
\end{aligned}
$$

Since $\left|{ }^{\varkappa_{2}} D_{q} \varphi\right|$ is preinvex, by Lemma 1 , we get

$$
\begin{aligned}
& \int_{0}^{\frac{1}{[2]_{q}}}\left|t-\frac{1}{[6]_{q}}\right|\left|\varkappa_{2} D_{q} \varphi\left(\varkappa_{2}+t \eta\left(\varkappa_{1}, \varkappa_{2}\right)\right)\right| d_{q} t \\
& \leq\left|\varkappa^{\varkappa_{2}} D_{q} \varphi\left(\varkappa_{1}\right)\right|\left(\frac{2 q^{2}[2]_{q}^{2}+[6]_{q}^{2}\left([6]_{q}-[3]_{q}\right)}{[2]_{q}^{3}[3]_{q}[6]_{q}^{3}}\right) \\
& \quad+\left|\varkappa^{\varkappa_{2}} D_{q} \varphi\left(\varkappa_{2}\right)\right|\left(2 \frac{q[3]_{q}[6]_{q}-q^{2}}{[2]_{q}[3]_{q}[6]_{q}^{3}}+\frac{1}{[2]_{q}^{3}}\left(\frac{q+q^{2}}{[3]_{q}}-\frac{q^{2}+2 q}{[6]_{q}}\right)\right) .
\end{aligned}
$$

Similarly, using the preinvexity of $\left|{ }^{\varkappa_{2}} D_{q} \varphi\right|$ and Lemma 1 , we have

$$
\begin{aligned}
\int_{\frac{1}{[2]_{q}}}^{1} \mid t & -\frac{[5]_{q}}{[6]_{q}}|| \varkappa_{2} D_{q} \varphi\left(\varkappa_{2}+\operatorname{t\eta }\left(\varkappa_{1}, \varkappa_{2}\right)\right) \mid d_{q} t \\
\leq & \left|\varkappa_{2} D_{q} \varphi\left(\varkappa_{1}\right)\right|\left(\frac{2 q^{2}[5]_{q}^{3}}{[2]_{q}[3]_{q}[6]_{q}^{3}}+\frac{[6]_{q}\left(1+[2]_{q}^{3}\right)-[3]_{q}[5]_{q}\left(1+[2]_{q}^{2}\right)}{[2]_{q}^{3}[3]_{q}[6]_{q}}\right) \\
& +\left|\varkappa^{2} D_{q} \varphi\left(\varkappa_{2}\right)\right|\left(2 \frac{q[5]_{q}^{2}[6]_{q}[3]_{q}-q^{2}[5]_{q}^{3}}{[2]_{q}[3]_{q}[6]_{q}^{3}}+\frac{q^{2}}{[2]_{q}[3]_{q}}-\frac{q[5]_{q}}{[2]_{q}[6]_{q}}\right. \\
& \left.-\frac{1}{[2]_{q}^{3}}\left[\frac{[5]_{q}\left(2 q+q^{2}\right)}{[6]_{q}}-\frac{q+q^{2}}{[3]_{q}}\right]\right) .
\end{aligned}
$$

By putting (4.13) and (4.14) in (4.12), we obtain the inequality (4.11), which finishes the proof.

Corollary 1 In Theorem 4 , if we take the limit $q \rightarrow 1^{-}$, then we obtain the following Simpson type inequality:

$$
\begin{aligned}
& \mid \frac{1}{\eta\left(\varkappa_{2}, \varkappa_{1}\right)} \int_{\varkappa_{2}+\eta\left(\varkappa_{1}, \varkappa_{2}\right)}^{\varkappa_{2}} \varphi(\varkappa) d \varkappa \\
& \quad-\frac{1}{6}\left[\varphi\left(\varkappa_{2}+\eta\left(\varkappa_{1}, \varkappa_{2}\right)\right)+4 \varphi\left(\frac{2 \varkappa_{2}+\eta\left(\varkappa_{1}, \varkappa_{2}\right)}{2}\right)+\varphi\left(\varkappa_{2}\right)\right] \mid \\
& \quad \leq \frac{5 \eta\left(\varkappa_{2}, \varkappa_{1}\right)}{72}\left[\left|\varphi^{\prime}\left(\varkappa_{1}\right)\right|+\left|\varphi^{\prime}\left(\varkappa_{2}\right)\right|\right],
\end{aligned}
$$

which can be viewed as a special case of the inequality derived in [34]. 
Therefore, we can deduce the following results for convex functions.

Remark 5 If we set $\eta\left(\varkappa_{2}, \varkappa_{1}\right)=\varkappa_{2}-\varkappa_{1}$ and $\eta\left(\varkappa_{1}, \varkappa_{2}\right)=\varkappa_{1}-\varkappa_{2}$ in Theorem 4, then we have the following inequality:

$$
\begin{aligned}
& \mid \frac{1}{\left(\varkappa_{2}-\varkappa_{1}\right)} \int_{\varkappa_{1}}^{\varkappa_{2}} \varphi\left(\varkappa^{\varkappa_{2}} d_{q} \varkappa-\frac{1}{[6]_{q}}\left[\varphi\left(\varkappa_{1}\right)+q^{2}[4]_{q} \varphi\left(\frac{\varkappa_{1}+q \varkappa_{2}}{[2]_{q}}\right)+q \varphi\left(\varkappa_{2}\right)\right] \mid\right. \\
& \leq q\left(\varkappa_{2}-\varkappa_{1}\right)\left\{\varkappa^{\varkappa_{2}} D_{q} \varphi\left(\varkappa_{1}\right)\left|\left[A_{1}(q)+A_{2}(q)\right]+\right| \varkappa^{\varkappa_{2}} D_{q} \varphi\left(\varkappa_{2}\right) \mid\left[B_{1}(q)+B_{2}(q)\right]\right\},
\end{aligned}
$$

which is given by Ali et al. [33].

Remark 6 If we set $\eta\left(\varkappa_{2}, \varkappa_{1}\right)=\varkappa_{2}-\varkappa_{1}, \eta\left(\varkappa_{1}, \varkappa_{2}\right)=\varkappa_{1}-\varkappa_{2}$, and $q \rightarrow 1^{-}$in Theorem 4, then Theorem 4 reduces to [26, Corollary 1$]$.

Remark 7 In Theorem 4, if $\eta\left(\varkappa_{2}, \varkappa_{1}\right)=\varkappa_{2}-\varkappa_{1}, \eta\left(\varkappa_{1}, \varkappa_{2}\right)=\varkappa_{1}-\varkappa_{2}, \varphi\left(\varkappa_{1}\right)=\varphi\left(\frac{\varkappa_{1}+\varkappa_{2}}{2}\right)=$ $\varphi\left(\varkappa_{2}\right)$, and $q \rightarrow 1^{-}$, then Theorem 4 reduces to [26, Corollary 3$]$.

The corresponding version of Simpson's inequality for powers in terms of the first $q$ derivative is incorporated in the following result.

Theorem 5 We assume that the assumptions of Lemma 2 hold. If $\left.\left.\right|^{\varkappa_{2}} D_{q} \varphi\right|^{p_{1}}$ is preinvex and integrable on I, where $p_{1}>1$ with $\frac{1}{r_{1}}+\frac{1}{p_{1}}=1$, then we have the following inequality:

$$
\begin{aligned}
& \mid \frac{1}{\eta\left(\varkappa_{2}, \varkappa_{1}\right)} \int_{\varkappa_{2}+\eta\left(\varkappa_{1}, \varkappa_{2}\right)}^{\varkappa_{2}} \varphi(\varkappa)^{\varkappa_{2}} d_{q} \varkappa \\
& -\frac{1}{[6]_{q}}\left[\varphi\left(\varkappa_{2}+\eta\left(\varkappa_{1}, \varkappa_{2}\right)\right)+[4]_{q} q^{2} \varphi\left(\frac{[2]_{q} \varkappa_{2}+\eta\left(\varkappa_{1}, \varkappa_{2}\right)}{[2]_{q}}\right)+q \varphi\left(\varkappa_{2}\right)\right] \mid \\
& \leq q \eta\left(\varkappa_{2}, \varkappa_{1}\right)\left[\left(\frac{q^{2 r_{1}}[4]_{q}^{r_{1}}}{[2]_{q}^{r_{1}+1}[6]_{q}^{r_{1}}}\right)^{\frac{1}{r_{1}}}\right. \\
& \times\left(\left.\left.\frac{1}{[2]_{q}^{3}}\right|^{\varkappa_{2}} D_{q} \varphi\left(\varkappa_{1}\right)\right|^{p_{1}}+\frac{q^{2}+2 q}{[2]_{q}^{3}}\left|\varkappa_{2} D_{q} \varphi\left(\varkappa_{2}\right)\right|^{p_{1}}\right)^{\frac{1}{p_{1}}} \\
& +\left(\frac{[2]_{q}^{r_{1}+1}[5]_{q}^{r_{1}}-q^{r_{1}}[4]_{q}^{r_{1}}}{[2]_{q}^{r_{1}+1}[6]_{q}^{r_{1}}}\right)^{\frac{1}{r_{1}}} \\
& \left.\times\left(\left.\left.\frac{q^{2}+2 q}{[2]_{q}^{3}}\right|^{\varkappa_{2}} D_{q} \varphi\left(\varkappa_{1}\right)\right|^{p_{1}}+\frac{q^{3}+q^{2}-q}{[2]_{q}^{3}}\left|\varkappa^{\varkappa_{2}} D_{q} \varphi\left(\varkappa_{2}\right)\right|^{p_{1}}\right)^{\frac{1}{p_{1}}}\right],
\end{aligned}
$$

where $0<q<1$.

Proof From the integrals in the right side of (4.12) and applying the well-known quantum Hölder integral inequality, it is found that

$$
\begin{aligned}
& \mid \frac{1}{\eta\left(\varkappa_{2}, \varkappa_{1}\right)} \int_{\varkappa_{2}+\eta\left(\varkappa_{1}, \varkappa_{2}\right)}^{\varkappa_{2}} \varphi\left(\varkappa^{\varkappa_{2}} d_{q} \varkappa\right. \\
& \quad-\frac{1}{[6]_{q}}\left[\varphi\left(\varkappa_{2}+\eta\left(\varkappa_{1}, \varkappa_{2}\right)\right)+[4]_{q} q^{2} \varphi\left(\frac{[2]_{q} \varkappa_{2}+\eta\left(\varkappa_{1}, \varkappa_{2}\right)}{[2]_{q}}\right)+q \varphi\left(\varkappa_{2}\right)\right] \mid
\end{aligned}
$$




$$
\begin{aligned}
& \leq q \eta\left(\varkappa_{2}, \varkappa_{1}\right)\left[\left(\int_{0}^{\frac{1}{[2]_{q}}}\left|t-\frac{1}{[6]_{q}}\right|^{r_{1}} d_{q} t\right)^{\frac{1}{r_{1}}}\right. \\
& \left.\times\left(\int_{0}^{\frac{1}{[2] q}}\left|\varkappa_{2} D_{q} \varphi\left(\varkappa_{2}+\eta\left(\varkappa_{1}, \varkappa_{2}\right)\right)\right|^{p_{1}} d_{q} t\right)^{\frac{1}{p_{1}}}\right] \\
& \times\left[+q \eta\left(\varkappa_{2}, \varkappa_{1}\right)\left(\int_{\frac{1}{[2]_{q}}}^{1}\left|t-\frac{[5]_{q}}{[6]_{q}}\right|^{r_{1}} d_{q} t\right)^{\frac{1}{r_{1}}}\right. \\
& \left.\times\left(\int_{\frac{1}{[2]_{q}}}^{1}\left|\varkappa_{2} D_{q} \varphi\left(\varkappa_{2}+\eta\left(\varkappa_{1}, \varkappa_{2}\right)\right)\right|^{p_{1}} d_{q} t\right)^{\frac{1}{p_{1}}}\right] .
\end{aligned}
$$

By using the preinvexity of $\left.\left.\right|^{\varkappa_{2}} D_{q} \varphi\right|^{p_{1}}$, we obtain

$$
\begin{aligned}
& \left|\frac{1}{\left(\varkappa_{2}-\varkappa_{1}\right)} \int_{\varkappa_{1}}^{\varkappa_{2}} \varphi(t)^{\varkappa_{2}} d_{q} t-\frac{1}{[6]_{q}}\left[\varphi\left(\varkappa_{1}\right)+q^{2}[4]_{q} \varphi\left(\frac{\varkappa_{1}+q \varkappa_{2}}{[2]_{q}}\right)+q \varphi\left(\varkappa_{2}\right)\right]\right| \\
& \leq q \eta\left(\varkappa_{2}, \varkappa_{1}\right)\left[\left(\int_{0}^{\frac{1}{22]_{q}}}\left|t-\frac{1}{[6]_{q}}\right|^{r_{1}} d_{q} t\right)^{\frac{1}{r_{1}}}\right. \\
& \left.\quad \times\left(\left|\varkappa_{2} D_{q} \varphi\left(\varkappa_{1}\right)\right|^{p_{1}} \int_{0}^{\frac{1}{[2]_{q}}} t d_{q} t+\left.\left.\right|^{\varkappa_{2}} D_{q} \varphi\left(\varkappa_{2}\right)\right|^{p_{1}} \int_{0}^{\frac{1}{[2]_{q}}}(1-t) d_{q} t\right)^{\frac{1}{p_{1}}}\right] \\
& +q \eta\left(\varkappa_{2}, \varkappa_{1}\right)\left[\left(\int_{\frac{1}{[2]_{q}}}^{1}\left|t-\frac{[5]_{q}}{[6]_{q}}\right|^{r_{1}} d_{q} t\right)^{\frac{1}{r_{1}}}\right. \\
& \left.\quad \times\left(\left|\varkappa_{2} D_{q} \varphi\left(\varkappa_{1}\right)\right|^{p_{1}} \int_{\frac{1}{[2]_{q}}}^{1} t d_{q} t+\left|\varkappa^{\varkappa_{2}} D_{q} \varphi\left(\varkappa_{2}\right)\right|^{p_{1}} \int_{\frac{1}{[2]_{q}}}^{1}(1-t) d_{q} t\right)^{\frac{1}{p_{1}}}\right] .
\end{aligned}
$$

To calculate the integrals in the right side of (4.16), if we first use the definition of the quantum integral, then we obtain

$$
\begin{aligned}
& \int_{0}^{\frac{1}{[2]_{q}}}\left|t-\frac{1}{[6]_{q}}\right|^{r_{1}} d_{q} t=\frac{1-q}{[2]_{q}} \sum_{\varkappa_{2}=0}^{\infty} q^{\varkappa_{2}}\left|\frac{q^{\varkappa_{2}}}{[2]_{q}}-\frac{1}{[6]_{q}}\right|^{r_{1}} \\
& \leq \frac{1-q}{[2]_{q}} \sum_{\varkappa_{2}=0}^{\infty} q^{\varkappa_{2}}\left|\frac{1}{[2]_{q}}-\frac{1}{[6]_{q}}\right|^{r_{1}} \\
& =\left(\frac{1}{[2]_{q}}-\frac{1}{[6]_{q}}\right)^{r_{1}} \frac{1}{[2]_{q}} \\
& =\frac{q^{2 r_{1}}[4]_{q}^{r_{1}}}{[2]_{q}^{r_{1}+1}[6]_{q}^{r_{1}}} \text {. }
\end{aligned}
$$

Similarly, we have

$$
\int_{\frac{1}{[2]_{q}}}^{1}\left|t-\frac{[5]_{q}}{[6]_{q}}\right|^{r_{1}} d_{q} t \leq \frac{[2]_{q}^{r_{1}+1}[5]_{q}^{r_{1}}-q^{r_{1}}[4]_{q}^{r_{1}}}{[2]_{q}^{r_{1}+1}[6]_{q}^{r_{1}}} .
$$


For the other integrals in the right side of (4.16) we consider the case when $\varkappa_{1}=0$ of Lemma 1 , we find that

$$
\begin{aligned}
& \int_{0}^{\frac{1}{[2]_{q}}} t d_{q} t=\frac{1}{[2]_{q}^{3}}, \\
& \int_{0}^{\frac{1}{[2]_{q}}}(1-t) d_{q} t=\frac{q^{2}+2 q}{[2]_{q}^{3}} .
\end{aligned}
$$

Similarly, we get

$$
\begin{aligned}
& \int_{\frac{1}{[2]_{q}}}^{1} t d_{q} t=\frac{q^{2}+2 q}{\left([2]_{q}\right)^{3}} \\
& \int_{\frac{1}{[2]_{q}}}^{1}(1-t) d_{q} t=\frac{q^{3}+q^{2}-q}{\left([2]_{q}\right)^{3}} .
\end{aligned}
$$

By substituting (4.17)-(4.22) in (4.16), we obtain the desired inequality (4.15), which completes the proof.

Remark 8 If we set $\eta\left(\varkappa_{2}, \varkappa_{1}\right)=\varkappa_{2}-\varkappa_{1}$ and $\eta\left(\varkappa_{1}, \varkappa_{2}\right)=\varkappa_{1}-\varkappa_{2}$ in Theorem 5 , then we have the following inequality:

$$
\begin{aligned}
& \left|\frac{1}{\left(\varkappa_{2}-\varkappa_{1}\right)} \int_{\varkappa_{1}}^{\varkappa_{2}} \varphi(\varkappa)^{\varkappa_{2}} d_{q} \varkappa-\frac{1}{[6]_{q}}\left[\varphi\left(\varkappa_{1}\right)+q^{2}[4]_{q} \varphi\left(\frac{\varkappa_{1}+q \varkappa_{2}}{[2]_{q}}\right)+q \varphi\left(\varkappa_{2}\right)\right]\right| \\
& \leq q\left(\varkappa_{2}-\varkappa_{1}\right)\left[\left(\frac{q^{2 r_{1}}[4]_{q}^{r_{1}}}{[2]_{q}^{r_{1}+1}[6]_{q}^{r_{1}}}\right)^{\frac{1}{r_{1}}}\right. \\
& \times\left(\left.\left.\frac{1}{[2]_{q}^{3}}\right|^{\varkappa_{2}} D_{q} \varphi\left(\varkappa_{1}\right)\right|^{p_{1}}+\frac{q^{2}+2 q}{[2]_{q}^{3}}\left|\varkappa^{\varkappa_{2}} D_{q} \varphi\left(\varkappa_{2}\right)\right|^{p_{1}}\right)^{\frac{1}{p_{1}}} \\
& +\left(\frac{[2]_{q}^{r_{1}+1}[5]_{q}^{r_{1}}-q^{r_{1}}[4]_{q}^{r_{1}}}{[2]_{q}^{r_{1}+1}[6]_{q}^{r_{1}}}\right)^{\frac{1}{r_{1}}} \\
& \left.\times\left(\left.\left.\frac{q^{2}+2 q}{[2]_{q}^{3}}\right|^{\varkappa_{2}} D_{q} \varphi\left(\varkappa_{1}\right)\right|^{p_{1}}+\frac{q^{3}+q^{2}-q}{[2]_{q}^{3}}\left|\varkappa_{2} D_{q} \varphi\left(\varkappa_{2}\right)\right|^{p_{1}}\right)^{\frac{1}{p_{1}}}\right],
\end{aligned}
$$

which is given by Ali et al. in [33].

Another version of Simpson's inequality for powers in terms of the first $q$-derivative is obtained as follows.

Theorem 6 Suppose that the assumptions of Lemma 2 hold. If $\left.\left.\right|^{\varkappa_{2}} D_{q} \varphi\right|^{p_{1}}$ is preinvex and integrable on $I$, where $p_{1}>1$, then we have the following inequality:

$$
\begin{aligned}
& \mid \frac{1}{\eta\left(\varkappa_{2}, \varkappa_{1}\right)} \int_{\varkappa_{2}+\eta\left(\varkappa_{1}, \varkappa_{2}\right)}^{\varkappa_{2}} \varphi\left(\varkappa^{\varkappa_{2}} d_{q} \varkappa\right. \\
& \quad-\frac{1}{[6]_{q}}\left[\varphi\left(\varkappa_{2}+\eta\left(\varkappa_{1}, \varkappa_{2}\right)\right)+[4]_{q} q^{2} \varphi\left(\frac{[2]_{q} \varkappa_{2}+\eta\left(\varkappa_{1}, \varkappa_{2}\right)}{[2]_{q}}\right)+q \varphi\left(\varkappa_{2}\right)\right] \mid
\end{aligned}
$$


Ali et al. Advances in Difference Equations

(2021) 2021:64

Page 13 of 21

$$
\begin{aligned}
& \leq q \eta\left(\varkappa_{2}, \varkappa_{1}\right)\left[\left(\frac{2 q}{[2]_{q}[6]_{q}^{2}}+\frac{q^{3}[3]_{q}-q}{[6]_{q}[2]_{q}^{3}}\right)^{1-\frac{1}{p_{1}}}\right. \\
& \times\left(\left.\left.A_{1}(q)\right|^{\varkappa_{2}} D_{q} \varphi\left(\varkappa_{1}\right)\right|^{p_{1}}+\left.\left.B_{1}(q)\right|^{\varkappa_{2}} D_{q} \varphi\left(\varkappa_{2}\right)\right|^{p_{1}}\right)^{\frac{1}{p_{1}}} \\
& +\left(2 q \frac{[5]_{q}^{2}}{[2]_{q}[6]_{q}^{2}}+\frac{1}{[2]_{q}}-\frac{[5]_{q}}{[6]_{q}}-\frac{[5]_{q}[2]_{q}^{2}-[6]_{q}}{[6]_{q}[2]_{q}^{3}}\right)^{1-\frac{1}{p_{1}}} \\
& \left.\times\left(\left.\left.A_{2}(q)\right|^{\varkappa_{2}} D_{q} \varphi\left(\varkappa_{1}\right)\right|^{p_{1}}+\left.\left.B_{2}(q)\right|^{\varkappa_{2}} D_{q} \varphi\left(\varkappa_{2}\right)\right|^{p_{1}}\right)^{\frac{1}{p_{1}}}\right] \text {, }
\end{aligned}
$$

where $0<q<1$ and $A_{1}(q), A_{2}(q), B_{1}(q), B_{2}(q)$ are given as in (4.1)-(4.4), respectively.

Proof Utilizing the results in the proof of Theorem 4, after applying the well-known power mean inequality to the integrals in the right side of (4.12), owing to the preinvexity of $\left.{ }^{\varkappa_{2}} D_{q} \varphi\right|^{p_{1}}$, we find that

$$
\begin{aligned}
& \mid \frac{1}{\eta\left(\varkappa_{2}, \varkappa_{1}\right)} \int_{\varkappa_{2}+\eta\left(\varkappa_{1}, \varkappa_{2}\right)}^{\varkappa_{2}} \varphi(\varkappa)^{\varkappa_{2}} d_{q} \varkappa \\
& -\frac{1}{[6]_{q}}\left[\varphi\left(\varkappa_{2}+\eta\left(\varkappa_{1}, \varkappa_{2}\right)\right)+[4]_{q} q^{2} \varphi\left(\frac{[2]_{q} \varkappa_{2}+\eta\left(\varkappa_{1}, \varkappa_{2}\right)}{[2]_{q}}\right)+q \varphi\left(\varkappa_{2}\right)\right] \mid \\
& \leq q \eta\left(\varkappa_{2}, \varkappa_{1}\right)\left[\left(\int_{0}^{\frac{1}{[2]_{q}}}\left|t-\frac{1}{[6]_{q}}\right| d_{q} t\right)^{1-\frac{1}{p_{1}}}\right. \\
& \times\left(\left|\varkappa_{2} D_{q} \varphi\left(\varkappa_{1}\right)\right|^{p_{1}} \int_{0}^{\frac{1}{[2]_{q}}} t\left|t-\frac{1}{[6]_{q}}\right| d_{q} t\right. \\
& \left.\left.+\left|\varkappa_{2} D_{q} \varphi\left(\varkappa_{2}\right)\right|^{p_{1}} \int_{0}^{\frac{1}{[2] q}}(1-t)\left|t-\frac{1}{[6]_{q}}\right| d_{q} t\right)^{\frac{1}{p_{1}}}\right] \\
& +q \eta\left(\varkappa_{2}, \varkappa_{1}\right)\left[\left(\int_{\frac{1}{[2]_{q}}}^{1}\left|t-\frac{[5]_{q}}{[6]_{q}}\right| d_{q} t\right)^{1-\frac{1}{p_{1}}}\right. \\
& \times\left(\left|\varkappa^{2} D_{q} \varphi\left(\varkappa_{1}\right)\right|^{p_{1}} \int_{\frac{1}{[2]_{q}}}^{1} t\left|t-\frac{[5]_{q}}{[6]_{q}}\right| d_{q} t\right. \\
& \left.\left.+\left|\varkappa_{2} D_{q} \varphi\left(\varkappa_{2}\right)\right|^{p_{1}} \int_{\frac{1}{[2]_{q}}}^{1}(1-t)\left|t-\frac{[5]_{q}}{[6]_{q}}\right| d_{q} t\right)^{\frac{1}{p_{1}}}\right] \\
& =q \eta\left(\varkappa_{2}, \varkappa_{1}\right)\left[\left(\int_{0}^{\frac{1}{[2]_{q}}}\left|t-\frac{1}{[6]_{q}}\right| d_{q} t\right)^{1-\frac{1}{p_{1}}}\right. \\
& \left.\times\left(\left.\left.A_{1}(q)\right|^{\varkappa_{2}} D_{q} \varphi\left(\varkappa_{1}\right)\right|^{p_{1}}+\left.\left.B_{1}(q)\right|^{\varkappa_{2}} D_{q} \varphi\left(\varkappa_{2}\right)\right|^{p_{1}}\right)^{\frac{1}{p_{1}}}\right] \\
& +\left[q \eta\left(\varkappa_{2}, \varkappa_{1}\right)\left(\int_{\frac{1}{[2]_{q}}}^{1}\left|t-\frac{[5]_{q}}{[6]_{q}}\right| d_{q} t\right)^{1-\frac{1}{p_{1}}}\right. \\
& \left.\times\left(\left.\left.A_{2}(q)\right|^{\varkappa_{2}} D_{q} \varphi\left(\varkappa_{1}\right)\right|^{p_{1}}+\left.\left.B_{2}(q)\right|^{\varkappa_{2}} D_{q} \varphi\left(\varkappa_{2}\right)\right|^{p_{1}}\right)^{\frac{1}{p_{1}}}\right] .
\end{aligned}
$$


We also observe that

$$
\begin{aligned}
\int_{0}^{\frac{1}{[2]_{q}}}\left|t-\frac{1}{[6]_{q}}\right| d_{q} t & =2 \int_{0}^{\frac{1}{[6]_{q}}}\left(\frac{1}{[6]_{q}}-t\right) d_{q} t+\int_{0}^{\frac{1}{[2]_{q}}}\left(t-\frac{1}{[6]_{q}}\right) d_{q} t \\
& =\frac{2 q}{[2]_{q}[6]_{q}^{2}}+\frac{q^{3}[3]_{q}-q}{[6]_{q}[2]_{q}^{3}}
\end{aligned}
$$

and by using similar operations, we have

$$
\int_{\frac{1}{[2]_{q}}}^{1}\left|t-\frac{[5]_{q}}{[6]_{q}}\right| d_{q} t=2 q \frac{[5]_{q}^{2}}{[2]_{q}[6]_{q}^{2}}+\frac{1}{[2]_{q}}-\frac{[5]_{q}}{[6]_{q}}-\frac{[5]_{q}[2]_{q}^{2}-[6]_{q}}{[6]_{q}[2]_{q}^{3}}
$$

By substituting (4.25) and (4.26) in (4.24), we obtain the required inequality (4.23). Hence, the proof is completed.

Corollary 2 If we take the limit $q \rightarrow 1^{-}$in Theorem 6 , then we have the following inequality:

$$
\begin{aligned}
\mid & \frac{1}{\eta\left(\varkappa_{2}, \varkappa_{1}\right)} \int_{\varkappa_{2}+\eta\left(\varkappa_{1}, \varkappa_{2}\right)}^{\varkappa_{2}} \varphi(\varkappa) d \varkappa \\
& -\frac{1}{6}\left[\varphi\left(\varkappa_{2}+\eta\left(\varkappa_{1}, \varkappa_{2}\right)\right)+4 \varphi\left(\frac{2 \varkappa_{2}+\eta\left(\varkappa_{1}, \varkappa_{2}\right)}{2}\right)+\varphi\left(\varkappa_{2}\right)\right] \mid \\
\leq & \frac{5^{1-\frac{1}{p_{1}}}}{72} \eta\left(\varkappa_{2}, \varkappa_{1}\right)\left[\left(\frac{29}{18}\left|\varphi^{\prime}\left(\varkappa_{1}\right)\right|^{p_{1}}+\frac{61}{18}\left|\varphi^{\prime}\left(\varkappa_{2}\right)\right|^{p_{1}}\right)^{\frac{1}{p_{1}}}\right. \\
& \left.+\left(\frac{61}{18}\left|\varphi^{\prime}\left(\varkappa_{1}\right)\right|^{p_{1}}+\frac{29}{18}\left|\varphi^{\prime}\left(\varkappa_{2}\right)\right|^{p_{1}}\right)^{\frac{1}{p_{1}}}\right],
\end{aligned}
$$

which can be viewed as a special case of the inequality derived in [34].

Remark 9 If we set $\eta\left(\varkappa_{2}, \varkappa_{1}\right)=\varkappa_{2}-\varkappa_{1}$ and $\eta\left(\varkappa_{1}, \varkappa_{2}\right)=\varkappa_{1}-\varkappa_{2}$ in Theorem 6 , then we have the following inequality:

$$
\begin{aligned}
& \left|\frac{1}{\left(\varkappa_{2}-\varkappa_{1}\right)} \int_{\varkappa_{1}}^{\varkappa_{2}} \varphi(\varkappa)^{\varkappa_{2}} d_{q} \varkappa-\frac{1}{[6]_{q}}\left[\varphi\left(\varkappa_{1}\right)+q^{2}[4]_{q} \varphi\left(\frac{\varkappa_{1}+q \varkappa_{2}}{[2]_{q}}\right)+q \varphi\left(\varkappa_{2}\right)\right]\right| \\
& \leq q\left(\varkappa_{2}-\varkappa_{1}\right)\left[\left(\frac{2 q}{[2]_{q}[6]_{q}^{2}}+\frac{q^{3}[3]_{q}-q}{[6]_{q}[2]_{q}^{3}}\right)^{1-\frac{1}{p_{1}}}\right. \\
& \times\left(\left.\left.A_{1}(q)\right|^{\varkappa_{2}} D_{q} \varphi\left(\varkappa_{1}\right)\right|^{p_{1}}+\left.\left.B_{1}(q)\right|^{\varkappa_{2}} D_{q} \varphi\left(\varkappa_{2}\right)\right|^{p_{1}}\right)^{\frac{1}{p_{1}}} \\
& +\left(2 q \frac{[5]_{q}^{2}}{[2]_{q}[6]_{q}^{2}}+\frac{1}{[2]_{q}}-\frac{[5]_{q}}{[6]_{q}}-\frac{[5]_{q}[2]_{q}^{2}-[6]_{q}}{[6]_{q}[2]_{q}^{3}}\right)^{1-\frac{1}{p_{1}}} \\
& \left.\times\left(\left.\left.A_{2}(q)\right|^{\varkappa_{2}} D_{q} \varphi\left(\varkappa_{1}\right)\right|^{p_{1}}+\left.\left.B_{2}(q)\right|^{\varkappa_{2}} D_{q} \varphi\left(\varkappa_{2}\right)\right|^{p_{1}}\right)^{\frac{1}{p_{1}}}\right],
\end{aligned}
$$

which is proved by Ali et al. in [33]. 
Remark 10 In Theorem 6, if we take $\eta\left(\varkappa_{2}, \varkappa_{1}\right)=\varkappa_{2}-\varkappa_{1}, \eta\left(\varkappa_{1}, \varkappa_{2}\right)=\varkappa_{1}-\varkappa_{2}$, and $q \rightarrow 1^{-}$, then we have the following inequality:

$$
\begin{aligned}
\left|\frac{1}{6}\left[\varphi\left(\varkappa_{1}\right)+4 \varphi\left(\frac{\varkappa_{1}+\varkappa_{2}}{2}\right)+\varphi\left(\varkappa_{2}\right)\right]-\frac{1}{\varkappa_{2}-\varkappa_{1}} \int_{\varkappa_{1}}^{\varkappa_{2}} \varphi(\varkappa) d \varkappa\right| \\
\leq \frac{5^{1-\frac{1}{p_{1}}}}{72}\left(\varkappa_{2}-\varkappa_{1}\right)\left[\left(\frac{29}{18}\left|\varphi^{\prime}\left(\varkappa_{1}\right)\right|^{p_{1}}+\frac{61}{18}\left|\varphi^{\prime}\left(\varkappa_{2}\right)\right|^{p_{1}}\right)^{\frac{1}{p_{1}}}\right. \\
\left.+\left(\frac{61}{18}\left|\varphi^{\prime}\left(\varkappa_{1}\right)\right|^{p_{1}}+\frac{29}{18}\left|\varphi^{\prime}\left(\varkappa_{2}\right)\right|^{p_{1}}\right)^{\frac{1}{p_{1}}}\right],
\end{aligned}
$$

which can be proved as a special case of inequality derived in [26].

\subsection{Newton's type inequalities}

In this subsection, we will present some quantum Newton's type inequalities for preinvex functions.

Theorem 7 We assume that the assumptions of Lemma 3 hold. If $\left.\right|^{\varkappa_{2}} D_{q} \varphi \mid$ is preinvex and integrable on $I$, then the following inequality holds for $q^{\varkappa_{2}}$-integrals:

$$
\begin{aligned}
& \mid \frac{1}{\eta\left(\varkappa_{2}, \varkappa_{1}\right)} \int_{\varkappa_{2}+\eta\left(\varkappa_{1}, \varkappa_{2}\right)}^{\varkappa_{2}} \varphi\left(\varkappa^{\varkappa_{2}} d_{q} \varkappa-\frac{1}{[8]_{q}}\left[\varphi\left(\varkappa_{2}+\eta\left(\varkappa_{1}, \varkappa_{2}\right)\right)\right.\right. \\
& \left.\quad+\frac{q^{3}[6]_{q}}{[2]_{q}} \varphi\left(\frac{[3]_{q} \varkappa_{2}+\eta\left(\varkappa_{1}, \varkappa_{2}\right)}{[3]_{q}}\right)+\frac{q^{2}[6]_{q}}{[2]_{q}} \varphi\left(\frac{[3]_{q} \varkappa_{2}+[2]_{q} \eta\left(\varkappa_{1}, \varkappa_{2}\right)}{[3]_{q}}\right)+q \varphi\left(\varkappa_{2}\right)\right] \mid \\
& \leq \\
& q \eta\left(\varkappa_{2}, \varkappa_{1}\right)\left\{\left|\varkappa_{2} D_{q} \varphi\left(\varkappa_{1}\right)\right|\left[A_{3}(q)+A_{4}(q)+A_{5}(q)\right]\right. \\
& \left.\quad+\left|{ }^{\varkappa_{2}} D_{q} \varphi\left(\varkappa_{2}\right)\right|\left[B_{3}(q)+B_{4}(q)+B_{5}(q)\right]\right\},
\end{aligned}
$$

where $0<q<1$ and $A_{3}(q), A_{4}(q), A_{5}(q), B_{3}(q), B_{4}(q), B_{5}(q)$ are given as in (4.5)-(4.10), respectively.

Proof Following arguments similar to those in the proof of Theorem 4, by taking into account Lemma 3, the desired inequality (4.27) can be obtained.

Corollary 3 If we take the limit $q \rightarrow 1^{-}$in Theorem 7 , then we have the following inequality:

$$
\begin{aligned}
& \mid \frac{1}{\eta\left(\varkappa_{2}, \varkappa_{1}\right)} \int_{\varkappa_{2}+\eta\left(\varkappa_{1}, \varkappa_{2}\right)}^{\varkappa_{2}} \varphi(\varkappa) d \varkappa \\
& \quad-\frac{1}{8}\left[\varphi\left(\varkappa_{2}+\eta\left(\varkappa_{1}, \varkappa_{2}\right)\right)+3 \varphi\left(\frac{3 \varkappa_{2}+\eta\left(\varkappa_{1}, \varkappa_{2}\right)}{3}\right)\right. \\
& \left.\quad+3 \varphi\left(\frac{3 \varkappa_{2}+2 \eta\left(\varkappa_{1}, \varkappa_{2}\right)}{3}\right)+\varphi\left(\varkappa_{2}\right)\right] \mid \\
& \leq \frac{25 \eta\left(\varkappa_{2}, \varkappa_{1}\right)}{576}\left[\left|\varphi^{\prime}\left(\varkappa_{1}\right)\right|+\left|\varphi^{\prime}\left(\varkappa_{2}\right)\right|\right],
\end{aligned}
$$

which can be viewed as a special case of the inequality given in [34]. 
Remark 11 If we set $\eta\left(\varkappa_{2}, \varkappa_{1}\right)=\varkappa_{2}-\varkappa_{1}$ and $\eta\left(\varkappa_{1}, \varkappa_{2}\right)=\varkappa_{1}-\varkappa_{2}$ in Theorem 7 , then we have the following inequality:

$$
\begin{aligned}
& \mid \frac{1}{\left(\varkappa_{2}-\varkappa_{1}\right)} \int_{\varkappa_{1}}^{\varkappa_{2}} \varphi(\varkappa)^{\varkappa_{2}} d_{q} \varkappa-\frac{1}{[8]_{q}}\left[\varphi\left(\varkappa_{1}\right)+\frac{q^{3}[6]_{q}}{[2]_{q}} \varphi\left(\frac{\varkappa_{1}+q[2]_{q} \varkappa_{2}}{[3]_{q}}\right)\right. \\
& \left.\quad+\frac{q^{2}[6]_{q}}{[2]_{q}} \varphi\left(\frac{[2]_{q} \varkappa_{1}+q^{2} \varkappa_{2}}{[3]_{q}}\right)+q \varphi\left(\varkappa_{2}\right)\right] \mid \\
& \leq q\left(\varkappa_{2}-\varkappa_{1}\right)\left\{\left|\varkappa_{2} D_{q} \varphi\left(\varkappa_{1}\right)\right|\left[A_{3}(q)+A_{4}(q)+A_{5}(q)\right]\right. \\
& \left.\quad+\left|\varkappa_{2} D_{q} \varphi\left(\varkappa_{2}\right)\right|\left[B_{3}(q)+B_{4}(q)+B_{5}(q)\right]\right\},
\end{aligned}
$$

which is proved by Ali et al. in [33].

Remark 12 If we set $\eta\left(\varkappa_{2}, \varkappa_{1}\right)=\varkappa_{2}-\varkappa_{1}, \eta\left(\varkappa_{1}, \varkappa_{2}\right)=\varkappa_{1}-\varkappa_{2}$, and $q \rightarrow 1^{-}$in Theorem 7 , then we have the following inequality:

$$
\begin{aligned}
& \mid \frac{1}{\varkappa_{2}-\varkappa_{1}} \int_{\varkappa_{1}}^{\varkappa_{2}} \varphi(\varkappa) d \varkappa \\
& \quad-\frac{1}{8}\left[\varphi\left(\varkappa_{1}\right)+3 \varphi\left(\frac{\varkappa_{1}+2 \varkappa_{2}}{3}\right)+3 \varphi\left(\frac{2 \varkappa_{1}+\varkappa_{2}}{3}\right)+\varphi\left(\varkappa_{2}\right)\right] \mid \\
& \quad \leq \frac{25\left(\varkappa_{2}-\varkappa_{1}\right)}{576}\left[\left|\varphi^{\prime}\left(\varkappa_{1}\right)\right|+\left|\varphi^{\prime}\left(\varkappa_{2}\right)\right|\right],
\end{aligned}
$$

which was derived as a special case of an inequality proved in [30].

Theorem 8 We assume that the assumptions of Lemma 3 hold. If $\left.\left.\right|^{\varkappa_{2}} D_{q} \varphi\right|^{p_{1}}$ is preinvex and integrable on $I$, where $p_{1}>1$ with $\frac{1}{r_{1}}+\frac{1}{p_{1}}=1$, then we have the following inequality:

$$
\begin{aligned}
& \mid \frac{1}{\eta\left(\varkappa_{2}, \varkappa_{1}\right)} \int_{\varkappa_{2}+\eta\left(\varkappa_{1}, \varkappa_{2}\right)}^{\varkappa_{2}} \varphi(\varkappa)^{\varkappa_{2}} d_{q} \varkappa-\frac{1}{[8]_{q}}\left[\varphi\left(\varkappa_{2}+\eta\left(\varkappa_{1}, \varkappa_{2}\right)\right)\right. \\
& +\frac{q^{3}[6]_{q}}{[2]_{q}} \varphi\left(\frac{[3]_{q} \varkappa_{2}+\eta\left(\varkappa_{1}, \varkappa_{2}\right)}{[3]_{q}}\right) \\
& \left.+\frac{q^{2}[6]_{q}}{[2]_{q}} \varphi\left(\frac{[3]_{q} \varkappa_{2}+[2]_{q} \eta\left(\varkappa_{1}, \varkappa_{2}\right)}{[3]_{q}}\right)+q \varphi\left(\varkappa_{2}\right)\right] \\
& \leq q \eta\left(\varkappa_{2}, \varkappa_{1}\right)\left(\frac{q^{3 r_{1}}[5]_{q}^{r_{1}}}{[3]_{q}^{r_{1}+1}[8]_{q}^{r_{1}}}\right)^{\frac{1}{r_{1}}} \\
& \times\left(\frac{1}{[3]_{q}^{2}[2]_{q}}\left|\varkappa_{2} D_{q} \varphi\left(\varkappa_{1}\right)\right|^{p_{1}}+\frac{[3]_{q}[2]_{q}-1}{[3]_{q}^{2}[2]_{q}}\left|\varkappa^{2} D_{q} \varphi\left(\varkappa_{2}\right)\right|^{p_{1}}\right)^{\frac{1}{p_{1}}} \\
& +q \eta\left(\varkappa_{2}, \varkappa_{1}\right)\left(\frac{q^{r_{1}}[2]_{q}-q^{2 r_{1}}}{[3]_{q}^{r_{1}+1}[2]_{q}^{r_{1}}}\right)^{\frac{1}{r_{1}}} \\
& \times\left(\frac{q^{2}+2}{[3]_{q}^{2}[2]_{q}}\left|\varkappa_{2} D_{q} \varphi\left(\varkappa_{1}\right)\right|^{p_{1}}+\frac{q[3]_{q}[2]_{q}-\left(q^{2}+2 q\right)}{[3]_{q}^{2}[2]_{q}}\left|\varkappa_{2} D_{q} \varphi\left(\varkappa_{2}\right)\right|^{p_{1}}\right)^{\frac{1}{p_{1}}} \\
& +q \eta\left(\varkappa_{2}, \varkappa_{1}\right)\left(\frac{q^{7 r_{1}}}{[8]_{q}^{r_{1}}}-\frac{[2]_{q}\left([7]_{q}[3]_{q}-[8]_{q}[2]_{q}\right)^{r_{1}}}{[8]_{q}^{r_{1}}[3]_{q}^{r_{1}+1}}\right)^{\frac{1}{r_{1}}}
\end{aligned}
$$




$$
\times\left(\left.\left.\frac{[3]_{q}^{2}-[2]_{q}^{2}}{[3]_{q}^{2}[2]_{q}}\right|^{\varkappa_{2}} D_{q} \varphi\left(\varkappa_{1}\right)\right|^{p_{1}}+\left.\left.\frac{q^{2}[3]_{q}[2]_{q}+[2]_{q}^{2}-[3]_{q}^{2}}{[3]_{q}^{2}[2]_{q}}\right|^{\varkappa_{2}} D_{q} \varphi\left(\varkappa_{2}\right)\right|^{p_{1}}\right)^{\frac{1}{p_{1}}}
$$

where $0<q<1$.

Proof If the techniques used in the proof of Theorem 5 are applied by taking into account the Lemma 3, the desired inequality (4.28) can be obtained.

Corollary 4 In Theorem 8 , if we take the limit $q \rightarrow 1^{-}$, then we have the following inequality:

$$
\begin{aligned}
\mid \frac{1}{\eta\left(\varkappa_{2}, \varkappa_{1}\right)} \int_{\varkappa_{2}+\eta\left(\varkappa_{1}, \varkappa_{2}\right)}^{\varkappa_{2}} \varphi(\varkappa) d \varkappa \\
\quad-\frac{1}{8}\left[\varphi\left(\varkappa_{1}+\eta\left(\varkappa_{1}, \varkappa_{2}\right)\right)+3 \varphi\left(\frac{3 \varkappa_{2}+\eta\left(\varkappa_{1}, \varkappa_{2}\right)}{3}\right)\right. \\
\left.\quad+3 \varphi\left(\frac{3 \varkappa_{2}+2 \eta\left(\varkappa_{1}, \varkappa_{2}\right)}{3}\right)+\varphi\left(\varkappa_{2}\right)\right] \mid \\
\leq \\
\quad+\frac{\eta\left(\varkappa_{2}, \varkappa_{1}\right)}{3}\left[\frac{5}{8}\left(\frac{\left|\varphi^{\prime}\left(\varkappa_{1}\right)\right|^{p_{1}}+5\left|\varphi^{\prime}\left(\varkappa_{2}\right)\right|^{p_{1}}}{6}\right)^{\frac{1}{p_{1}}}\right)^{\frac{1}{r_{1}}}\left(\frac{\left|\varphi^{\prime}\left(\varkappa_{1}\right)\right|^{p_{1}}+\left|\varphi^{\prime}\left(\varkappa_{2}\right)\right|^{p_{1}}}{2}\right)^{\frac{1}{p_{1}}} \\
\left.\quad+\left(\frac{3.7^{r_{1}}-2}{8^{r_{1}}}\right)^{\frac{1}{r_{1}}}\left(\frac{5\left|\varphi^{\prime}\left(\varkappa_{1}\right)\right|^{p_{1}}+\left|\varphi^{\prime}\left(\varkappa_{2}\right)\right|^{p_{1}}}{6}\right)^{\frac{1}{p_{1}}}\right] .
\end{aligned}
$$

Remark 13 If we set $\eta\left(\varkappa_{2}, \varkappa_{1}\right)=\varkappa_{2}-\varkappa_{1}$ and $\eta\left(\varkappa_{1}, \varkappa_{2}\right)=\varkappa_{1}-\varkappa_{2}$ in (4.29), then the inequality (4.29) reduces to the inequality presented in [12, Remark 4].

Remark 14 If we set $\eta\left(\varkappa_{2}, \varkappa_{1}\right)=\varkappa_{2}-\varkappa_{1}$ and $\eta\left(\varkappa_{1}, \varkappa_{2}\right)=\varkappa_{1}-\varkappa_{2}$ in Theorem 8 , then we have the following inequality:

$$
\begin{aligned}
& \mid \frac{1}{\left(\varkappa_{2}-\varkappa_{1}\right)} \int_{\varkappa_{1}}^{\varkappa_{2}} \varphi(\varkappa)^{\varkappa_{2}} d_{q} \varkappa-\frac{1}{[8]_{q}}\left[\varphi\left(\varkappa_{1}\right)+\frac{q^{3}[6]_{q}}{[2]_{q}} \varphi\left(\frac{\varkappa_{1}+q[2]_{q} \varkappa_{2}}{[3]_{q}}\right)\right. \\
& \left.+\frac{q^{2}[6]_{q}}{[2]_{q}} \varphi\left(\frac{[2]_{q} \varkappa_{1}+q^{2} \varkappa_{2}}{[3]_{q}}\right)+q \varphi\left(\varkappa_{2}\right)\right] \\
& \leq q(b-a)\left(\frac{q^{3 r_{1}}[5]_{q}^{r_{1}}}{[3]_{q}^{r_{1}+1}[8]_{q}^{r_{1}}}\right)^{\frac{1}{r_{1}}} \\
& \times\left(\left.\left.\frac{1}{[3]_{q}^{2}[2]_{q}}\right|^{\varkappa_{2}} D_{q} \varphi\left(\varkappa_{1}\right)\right|^{p_{1}}+\left.\left.\frac{[3]_{q}[2]_{q}-1}{[3]_{q}^{2}[2]_{q}}\right|^{\varkappa_{2}} D_{q} \varphi\left(\varkappa_{2}\right)\right|^{p_{1}}\right)^{\frac{1}{p_{1}}} \\
& +q(b-a)\left(\frac{q^{r_{1}}[2]_{q}-q^{2 r_{1}}}{[3]_{q}^{r_{1}+1}[2]_{q}^{r_{1}}}\right)^{\frac{1}{r_{1}}} \\
& \times\left(\frac{q^{2}+2}{[3]_{q}^{2}[2]_{q}}\left|\varkappa_{2} D_{q} \varphi\left(\varkappa_{1}\right)\right|^{p_{1}}+\left.\left.\frac{q[3]_{q}[2]_{q}-\left(q^{2}+2 q\right)}{[3]_{q}^{2}[2]_{q}}\right|^{\varkappa_{2}} D_{q} \varphi\left(\varkappa_{2}\right)\right|^{p_{1}}\right)^{\frac{1}{p_{1}}} \\
& +q(b-a)\left(\frac{q^{7 r_{1}}}{[8]_{q}^{r_{1}}}-\frac{[2]_{q}\left([7]_{q}[3]_{q}-[8]_{q}[2]_{q}\right)^{r_{1}}}{[8]_{q}^{r_{1}}[3]_{q}^{r_{1}+1}}\right)^{\frac{1}{r_{1}}}
\end{aligned}
$$




$$
\times\left(\left.\left.\frac{[3]_{q}^{2}-[2]_{q}^{2}}{[3]_{q}^{2}[2]_{q}}\right|^{\varkappa_{2}} D_{q} \varphi\left(\varkappa_{1}\right)\right|^{p_{1}}+\frac{q^{2}[3]_{q}[2]_{q}+[2]_{q}^{2}-[3]_{q}^{2}}{[3]_{q}^{2}[2]_{q}}\left|\varkappa_{2} D_{q} \varphi\left(\varkappa_{2}\right)\right|^{p_{1}}\right)^{\frac{1}{p_{1}}}
$$

which is given by Ali et al. in [33].

Theorem 9 Suppose that the assumptions of Lemma 3 hold. If $\left.\left.\right|^{\varkappa_{2}} D_{q} \varphi\right|^{p_{1}}$ is preinvex and integrable on I, where $p_{1}>1$, then we have the following inequality:

$$
\begin{aligned}
& \mid \frac{1}{\eta\left(\varkappa_{2}, \varkappa_{1}\right)} \int_{\varkappa_{2}+\eta\left(\varkappa_{1}, \varkappa_{2}\right)}^{\varkappa_{2}} \varphi(\varkappa)^{\varkappa_{2}} d_{q} \varkappa-\frac{1}{[8]_{q}}\left[\varphi\left(\varkappa_{2}+\eta\left(\varkappa_{1}, \varkappa_{2}\right)\right)\right. \\
& \left.+\frac{q^{3}[6]_{q}}{[2]_{q}} \varphi\left(\frac{[3]_{q} \varkappa_{2}+\eta\left(\varkappa_{1}, \varkappa_{2}\right)}{[3]_{q}}\right)+\frac{q^{2}[6]_{q}}{[2]_{q}} \varphi\left(\frac{[3]_{q} \varkappa_{2}+[2]_{q} \eta\left(\varkappa_{1}, \varkappa_{2}\right)}{[3]_{q}}\right)+q \varphi\left(\varkappa_{2}\right)\right] \mid \\
& \leq q \eta\left(\varkappa_{2}, \varkappa_{1}\right)\left[\left(\frac{2 q}{[8]_{q}^{2}[2]_{q}}+\frac{[8]_{q}-[3]_{q}[2]_{q}}{[3]_{q}^{2}[2]_{q}[8]_{q}}\right)^{1-\frac{1}{p_{1}}}\right. \\
& \times\left(\left.\left.A_{3}(q)\right|^{\varkappa_{2}} D_{q} \varphi\left(\varkappa_{1}\right)\right|^{p_{1}}+\left.\left.B_{3}(q)\right|^{\varkappa_{2}} D_{q} \varphi\left(\varkappa_{2}\right)\right|^{p_{1}}\right)^{\frac{1}{p_{1}}} \\
& +\left(\frac{2 q}{[2]_{q}^{3}}+\frac{q}{[3]_{q}^{2}[2]_{q}}+\frac{1-[3]_{q}[2]_{q}}{[3]_{q}^{2}[2]_{q}}\right)^{1-\frac{1}{p_{1}}} \\
& \times\left(\left.\left.A_{4}(q)\right|^{\varkappa_{2}} D_{q} \varphi\left(\varkappa_{1}\right)\right|^{p_{1}}+\left.\left.B_{4}(q)\right|^{\varkappa_{2}} D_{q} \varphi\left(\varkappa_{2}\right)\right|^{p_{1}}\right)^{\frac{1}{p_{1}}} \\
& +\left(2 \frac{q[7]_{q}^{2}}{[8]_{q}^{2}[2]_{q}}+\frac{[3]_{q}^{2}+[2]_{q}^{2}}{[2]_{q}[3]_{q}^{2}}-\frac{[7]_{q}\left([3]_{q}+[2]_{q}\right)}{[8]_{q}[3]_{q}}\right)^{1-\frac{1}{p_{1}}} \\
& \left.\times\left(\left.\left.A_{5}(q)\right|^{\varkappa_{2}} D_{q} \varphi\left(\varkappa_{1}\right)\right|^{p_{1}}+\left.\left.B_{5}(q)\right|^{\varkappa_{2}} D_{q} \varphi\left(\varkappa_{2}\right)\right|^{p_{1}}\right)^{\frac{1}{p_{1}}}\right],
\end{aligned}
$$

where $0<q<1$ and $A_{3}(q), A_{4}(q), A_{5}(q), B_{3}(q), B_{4}(q), B_{5}(q)$ are given as in (4.5)-(4.10), respectively.

Proof The proof follows along the same lines used in the proof of Theorem 6 by taking into account Lemma 3.

Corollary 5 In Theorem 9, if we take the limit $q \rightarrow 1^{-}$, then we have the following Newtontype inequality:

$$
\begin{aligned}
& \mid \frac{1}{\eta\left(\varkappa_{2}, \varkappa_{1}\right)} \int_{\varkappa_{2}+\eta\left(\varkappa_{1}, \varkappa_{2}\right)}^{\varkappa_{2}} \varphi(\varkappa) d \varkappa \\
& \quad-\frac{1}{8}\left[\varphi\left(\varkappa_{1}+\eta\left(\varkappa_{1}, \varkappa_{2}\right)\right)+3 \varphi\left(\frac{3 \varkappa_{2}+\eta\left(\varkappa_{1}, \varkappa_{2}\right)}{3}\right)\right. \\
& \left.\quad+3 \varphi\left(\frac{3 \varkappa_{2}+2 \eta\left(\varkappa_{1}, \varkappa_{2}\right)}{3}\right)+\varphi\left(\varkappa_{2}\right)\right] \mid \\
& \leq \frac{\eta\left(\varkappa_{2}, \varkappa_{1}\right)}{36}\left[\left(\frac{17}{16}\right)^{1-\frac{1}{p_{1}}}\left(\frac{251}{1152}\left|\varphi^{\prime}\left(\varkappa_{1}\right)\right|^{p_{1}}+\frac{973}{1152}\left|\varphi^{\prime}\left(\varkappa_{2}\right)\right|^{p_{1}}\right)^{\frac{1}{p_{1}}}\right. \\
& \quad+\left(\frac{\left|\varphi^{\prime}\left(\varkappa_{1}\right)\right|^{p_{1}}+\left|\varphi^{\prime}\left(\varkappa_{2}\right)\right|^{p_{1}}}{2}\right)^{\frac{1}{p_{1}}}
\end{aligned}
$$




$$
\left.+\left(\frac{17}{16}\right)^{1-\frac{1}{p_{1}}}\left(\frac{973}{1152}\left|\varphi^{\prime}\left(\varkappa_{1}\right)\right|^{p_{1}}+\frac{251}{1152}\left|\varphi^{\prime}\left(\varkappa_{2}\right)\right|^{p_{1}}\right)^{\frac{1}{p_{1}}}\right]
$$

Remark 15 If we set $\eta\left(\varkappa_{2}, \varkappa_{1}\right)=\varkappa_{2}-\varkappa_{1}$ and $\eta\left(\varkappa_{1}, \varkappa_{2}\right)=\varkappa_{1}-\varkappa_{2}$ in (4.31), then the inequality (4.31) reduces to the inequality presented in [12, Remark 5].

Remark 16 If we set $\eta\left(\varkappa_{2}, \varkappa_{1}\right)=\varkappa_{2}-\varkappa_{1}$ and $\eta\left(\varkappa_{1}, \varkappa_{2}\right)=\varkappa_{1}-\varkappa_{2}$ in Theorem 9, then we have the following inequality:

$$
\begin{aligned}
& \mid \frac{1}{\left(\varkappa_{2}-\varkappa_{1}\right)} \int_{\varkappa_{1}}^{\varkappa_{2}} \varphi(\varkappa)^{\varkappa_{2}} d_{q} \varkappa-\frac{1}{[8]_{q}}\left[\varphi\left(\varkappa_{1}\right)+\frac{q^{3}[6]_{q}}{[2]_{q}} \varphi\left(\frac{\varkappa_{1}+q[2]_{q} \varkappa_{2}}{[3]_{q}}\right)\right. \\
& \left.+\frac{q^{2}[6]_{q}}{[2]_{q}} \varphi\left(\frac{[2]_{q} \varkappa_{1}+q^{2} \varkappa_{2}}{[3]_{q}}\right)+q \varphi\left(\varkappa_{2}\right)\right] \\
& \leq q\left(\varkappa_{2}-\varkappa_{1}\right)\left[\left(\frac{2 q}{[8]_{q}^{2}[2]_{q}}+\frac{[8]_{q}-[3]_{q}[2]_{q}}{[3]_{q}^{2}[2]_{q}[8]_{q}}\right)^{1-\frac{1}{p_{1}}}\right. \\
& \times\left(\left.\left.A_{3}(q)\right|^{\varkappa_{2}} D_{q} \varphi\left(\varkappa_{1}\right)\right|^{p_{1}}+\left.\left.B_{3}(q)\right|^{\varkappa_{2}} D_{q} \varphi\left(\varkappa_{2}\right)\right|^{p_{1}}\right)^{\frac{1}{p_{1}}} \\
& +\left(\frac{2 q}{[2]_{q}^{3}}+\frac{q}{[3]_{q}^{2}[2]_{q}}+\frac{1-[3]_{q}[2]_{q}}{[3]_{q}^{2}[2]_{q}}\right)^{1-\frac{1}{p_{1}}} \\
& \times\left(\left.\left.A_{4}(q)\right|^{\varkappa_{2}} D_{q} \varphi\left(\varkappa_{1}\right)\right|^{p_{1}}+\left.\left.B_{4}(q)\right|^{\varkappa_{2}} D_{q} \varphi\left(\varkappa_{2}\right)\right|^{p_{1}}\right)^{\frac{1}{p_{1}}} \\
& +\left(2 \frac{q[7]_{q}^{2}}{[8]_{q}^{2}[2]_{q}}+\frac{[3]_{q}^{2}+[2]_{q}^{2}}{[2]_{q}[3]_{q}^{2}}-\frac{[7]_{q}\left([3]_{q}+[2]_{q}\right)}{[8]_{q}[3]_{q}}\right)^{1-\frac{1}{p_{1}}} \\
& \left.\times\left(\left.\left.A_{5}(q)\right|^{\varkappa_{2}} D_{q} \varphi\left(\varkappa_{1}\right)\right|^{p_{1}}+\left.\left.B_{5}(q)\right|^{\varkappa_{2}} D_{q} \varphi\left(\varkappa_{2}\right)\right|^{p_{1}}\right)^{\frac{1}{p_{1}}}\right],
\end{aligned}
$$

which is derived by Ali et al. in [33].

\section{Conclusion}

In this paper, we proved some new inequalities of Simpson's and Newton's type for $q$ differentiable preinvex functions by using the notion of $q^{\varkappa_{2}}$-quantum integral. It is also shown that some classical results can be obtained by the results presented in the current research by taking the limit $q \rightarrow 1^{-}$. It will be an interesting problem to prove similar inequalities for the functions of two variables.

\section{Acknowledgements}

The authors would like to express their sincere thanks to the editor and the anonymous reviewers for their helpful comments and suggestions.

Funding

The work was supported by the Natural Science Foundation of China (Grant Nos. 61673169, 11301127, 11701176 11626101, 11601485, 11971241).

Availability of data and materials

Not applicable.

Competing interests

The authors declare that they have no competing interests. 


\section{Author details}

1 Jiangsu Key Laboratory for NSLSCS, School of Mathematical Sciences, Nanjing Normal University, Nanjing, China.

${ }^{2}$ Department of Mathematics, Government College University, Lahore, Pakistan. ${ }^{3}$ Department of Mathematics, Faculty of Science and Arts, Düzce University, Düzce, Turkey. ${ }^{4}$ Department of Mathematics, Anand International College of Engineering, Jaipur, India. ${ }^{5}$ Department of Mathematics, University of Management Technology, Lahore, Pakistan. ${ }^{6}$ Department of Mathematics, Huzhou University, Huzhou, China.

\section{Publisher's Note}

Springer Nature remains neutral with regard to jurisdictional claims in published maps and institutional affiliations.

Received: 26 November 2020 Accepted: 7 January 2021 Published online: 21 January 2021

\section{References}

1. Ernst, T:: A Comprehensive Treatment of Q-calculus. Springer, Berlin (2012)

2. Ernst, T.: The History of Q-calculus and a New Method. Citeseer, Sweden (2000)

3. Gauchman, H.: Integral inequalities in q-calculus. Comput. Math. Appl. 47(2-3), 281-300 (2004)

4. Jackson, D.O., Fukuda, T., Dunn, O., Majors, E.: On q-definite integrals. In: Quart. J. Pure Appl. Math (1910) Citeseer

5. Kac, V., Cheung, P.: Quantum Calculus. Springer, Berlin (2001)

6. Agarwal, R.P.: A propos d'une note de M. Pierre Humbert. C. R. Acad. Sci. Paris 236(21), 2031-2032 (1953)

7. Al-Salam, W.A.: Some fractional q-integrals and q-derivatives. Proc. Edinb. Math. Soc. 15(2), 135-140 (1966)

8. Rajkovic, P.M., Stankovic, M.S., Marinkovic, S.D., et al.: The zeros of polynomials orthogonal with respect to $q$-integral on several intervals in the complex plane. In: Proceedings of the Fifth International Conference on Geometry, Integrability and Quantization, pp. 178-188. Institute of Biophysics and Biomedical Engineering, Bulgarian Academy of Sciences, Sofia (2004)

9. Alp, N., Sarıkaya, M.Z., Kunt, M., Işscan, ì.: q-Hermite Hadamard inequalities and quantum estimates for midpoint type inequalities via convex and quasi-convex functions. J. King Saud Univ., Sci. 30(2), 193-203 (2018)

10. Bin-Mohsin, B., Awan, M.U., Noor, M.A., Riahi, L., Noor, K.I., Almutairi, B.: New quantum Hermite-Hadamard inequalities utilizing harmonic convexity of the functions. IEEE Access 7, 20479-20483 (2019)

11. Budak, H., Ali, M.A., Tarhanaci, M.: Some new quantum Hermite-Hadamard-like inequalities for coordinated convex functions. J. Optim. Theory Appl. 186, 899-910 (2020)

12. Budak, H., Erden, S., Ali, M.A.: Simpson and Newton type inequalities for convex functions via newly defined quantum integrals. Math. Methods Appl. Sci. 44(1), 378-390 (2021)

13. Deng, Y., Awan, M.U., Wu, S.: Quantum integral inequalities of Simpson-type for strongly preinvex functions. Mathematics 7(8), 751 (2019)

14. Jhanthanam, S., Tariboon, J., Ntouyas, S.K., Nonlaopon, K.: On q-Hermite-Hadamard inequalities for differentiable convex functions. Mathematics 7(7), 632 (2019)

15. Khan, M.A., Mohammad, N., Nwaeze, E.R., Chu, Y.-M.: Quantum Hermite-Hadamard inequality by means of a Green function. Adv. Differ. Equ. 2020(1), 1 (2020)

16. Liu, W., Zhuang, H.: Some quantum estimates of Hermite-Hadamard inequalities for convex functions. J. Appl. Anal. Comput. 7, 501-522 (2016)

17. Noor, M.A., Awan, M.U., Noor, K.I.: Quantum Ostrowski inequalities for q-differentiable convex functions. J. Math. Inequal. 10(4), 1013-1018 (2016)

18. Noor, M.A., Noor, K.I., Awan, M.U.: Some quantum estimates for Hermite-Hadamard inequalities. Appl. Math. Comput. 251, 675-679 (2015)

19. Noor, M.A., Noor, K.I., Awan, M.U.: Some quantum integral inequalities via preinvex functions. Appl. Math. Comput. 269, 242-251 (2015)

20. Nwaeze, E.R., Tameru, A.M.: New parameterized quantum integral inequalities via $\eta$-quasiconvexity. Adv. Differ. Equ. 2019(1), 425 (2019)

21. Tunç, M., Göv, E., Balgeçti, S.: Simpson type quantum integral inequalities for convex functions. Miskolc Math. Notes 19(1), 649-664 (2018)

22. Vivas-Cortez, M., Aamir Ali, M., Kashuri, A., Bashir Sial, I., Zhang, Z.: Some new Newton's type integral inequalities for co-ordinated convex functions in quantum calculus. Symmetry 12(9), 1476 (2020)

23. Zhang, Y., Du, T.-S., Wang, H., Shen, Y.-J.: Different types of quantum integral inequalities via $(\alpha, m)$-convexity. J. Inequal. Appl. 2018(1), 264 (2018)

24. Zhuang, H., Liu, W., Park, J.: Some quantum estimates of Hermite-Hadmard inequalities for quasi-convex functions. Miskolc Math. Notes 17, 2 (2016)

25. Dragomir, S.S., Agarwal, R., Cerone, P.: On Simpson's inequality and applications. RGMIA Res. Rep. Collect. 2(3), (1999)

26. Alomari, M., Darus, M., Dragomir, S.S.: New inequalities of simpson's type for s-convex functions with applications. Res Rep. Collect. 12(4) (2009)

27. Sarikaya, M.Z., Set, E., Ozdemir, M.E.: On new inequalities of Simpson's type for s-convex functions. Comput. Math. Appl. 60(8), 2191-2199 (2010)

28. Noor, M., Noor, K., Iftikhar, S.: Some Newton's type inequalities for harmonic convex functions. J. Adv. Math. Stud. 9(1), 7-16 (2016)

29. Noor, M.A., Noor, K.I., Iftikhar, S.: Newton inequalities for p-harmonic convex functions. Honam Math. J. 40(2), 239-250 (2018)

30. Iftikhar, S., Kumam, P., Erden, S.: Newton's type integral inequalities via local fractional integrals. Fractals 28(3), 2050037 (2020)

31. Tariboon, J., Ntouyas, S.K.: Quantum calculus on finite intervals and applications to impulsive difference equations. Adv. Differ. Equ. 2013(1), 282 (2013)

32. Bermudo, S., Kórus, P. Valdés, J.N.: On q-Hermite-Hadamard inequalities for general convex functions. Acta Math Hung. 162, 364-374 (2020) 
33. Ali, M.A., Chu, Y.-M., Budak, H., Akkurt, A., Yıldırım, H., Zahid, M.A.: Quantum variant of Montgomery identity and Ostrowski-type inequalities for the mappings of two variables. Adv. Differ. Equ. 2021, Article ID 25 (2021)

34. Du, T.-S., Liao, J.-G., Li, Y.-J.: Properties and integral inequalities of Hadamard-Simpson type for the generalized $(s, m)$-preinvex functions. J. Nonlinear Sci. Appl. 9(5), 3112-3126 (2016)

Submit your manuscript to a SpringerOpen ${ }^{\circ}$ journal and benefit from:

- Convenient online submission

- Rigorous peer review

- Open access: articles freely available online

- High visibility within the field

- Retaining the copyright to your article

Submit your next manuscript at $\gg$ springeropen.com 